\title{
Isosingular Sets and Deflation
}

\author{
Jonathan D. Hauenstein* Charles W. Wampler ${ }^{\dagger}$
}

June 23,2011

\begin{abstract}
This article introduces the concept of isosingular sets, which are irreducible algebraic subsets of the set of solutions to a system of polynomial equations that share a common singularity structure. The definition of these sets depends on deflation, a procedure that uses differentiation to regularize solutions. A weak form of deflation has proven useful in regularizing algebraic sets, making them amenable to treatment by the algorithms of numerical algebraic geometry. We introduce a strong form of deflation and define deflation sequences, which are similar to the sequences arising in Thom-Boardman singularity theory. We then define isosingular sets in terms of deflation sequences. We also define the isosingular local dimension and examine the properties of isosingular sets. While isosingular sets are of theoretical interest as constructs for describing singularity structures of algebraic sets, they also expand the kinds of algebraic sets that can be investigated with methods from numerical algebraic geometry.

Key words and phrases. irreducible algebraic set, deflation, deflation sequence, multiplicity, isosingular set, isosingular point, isosingular local dimension, numerical algebraic geometry, polynomial system, witness point, witness set, local dimension.
\end{abstract}

2010 Mathematics Subject Classification. Primary 65H10; Secondary 13P05, 14Q99, $68 \mathrm{~W} 30$.

\section{Introduction}

The singularities of an algebraic set are of fundamental interest in algebraic geometry. This article introduces isosingular sets as a framework for a stratified decomposition of an algebraic set and its subsets of singular points. Our approach makes use of deflation, a recursive process that is used in numerical algebraic geometry to regularize algebraic sets that have generic multiplicity greater than one. We introduce a stronger variant of deflation that regularizes isosingular sets, both allowing these to be treated effectively by numerical methods and providing a theoretical construct for understanding singularities. Moreover, we show that the deflation methods of [10, 22 are related to constructions performed in Thom-Boardman singularity theory [1, 14].

In a sense that we will soon make explicit, the generic points of an irreducible algebraic set all have the same singularity structure, represented by a sequence of nonnegative integers that we

\footnotetext{
*Department of Mathematics, Mailstop 3368, Texas A\&M University, College Station, TX 77843 (jhauenst@math.tamu.edu, www.math.tamu.edu/ jhauenst). This author was supported by Texas A\&M University, the Mittag-Leffler Institute, and NSF grant DMS-0915211.

${ }^{\dagger}$ General Motors Research and Development, Mail Code 480-106-224, 30500 Mound Road, Warren, MI 48090 (Charles.W.Wampler@gm.com, www.nd.edu/ cwample1) This author was supported by the Mittag-Leffler Institute and NSF grant DMS-0712910.
} 
call the deflation sequence of the set. This deflation sequence is related to sequences which arise in the study of Thom-Boardman singularities for smooth mappings. This leads us to define isosingular sets as closures of sets of points in an algebraic set that have the same deflation sequence. Clearly, irreducible components are isosingular sets, but there may also exist proper subsets of an irreducible component that are isosingular with a different singularity structure than the containing component. In this manner, we obtain a stratification of isosingular sets that capture the singularity structure of an algebraic set.

We review basic concepts of Thom-Boardman singularities and a form of deflation based on [10, 22, which we call weak deflation, that is currently used in numerical algebraic geometry in Section 2 We define deflation sequences in Section 3 and show in Section 4 that there are three equivalent forms of a new deflation method, which we call strong deflation, that yield this sequence. The first is based on determinants, as in Thom-Boardman singularity theory, while the other two, which are more amenable for numerical computation, are developed by modifying weak deflation to make use of a matrix rank deficiency formulation from [2]. Isosingular sets and isosingular points are described in Section 5 Algorithms for computing deflation sequences and isosingular sets are described in Section 6. The last section, Section 7, presents a simple illustrative example and a more challenging example taken from kinematics.

\section{Background}

\subsection{Thom-Boardman singularities}

We review some basic concepts regarding Thom-Boardman singularities, of which further details can be found in 1, 14. Let $f: \mathcal{M}^{m} \rightarrow \mathcal{N}^{n}$ be a smooth mapping where $\mathcal{M}$ and $\mathcal{N}$ are manifolds. If $x \in \mathcal{M}^{m}$, let dnull $(f, x)$ be the dimension of the kernel of the differential, $D f$, of $f$ at $x$. The Thom-Boardman stratification of $f$ follows a recursive process as follows. Let $d \geq 0$ and define

$$
\Sigma^{d}(f)=\left\{x \in \mathcal{M}^{m} \mid \operatorname{dnull}(f, x)=d\right\} .
$$

Suppose that $i_{1}, \ldots, i_{k}, i_{k+1} \geq 0$ and that $\Sigma^{I}(f)=\Sigma^{i_{1}, \ldots, i_{k}}(f)$ is defined and is a manifold. Then, define

$$
\Sigma^{i_{1}, \ldots, i_{k}, i_{k+1}}(f)=\left\{x \in \Sigma^{I}(f) \mid \operatorname{dnull}\left(\left.f\right|_{\Sigma^{I}(f)}, x\right)=i_{k+1}\right\}
$$

where $\left.f\right|_{\Sigma^{I}(f)}$ is the restriction of $f$ to $\Sigma^{I}(f)$. In $[8$, Boardman presents a definition in terms of jet spaces, shows that $\Sigma^{I}(f)$ is a manifold for generic maps with respect to the Whitney topology on the set of smooth maps, and computes the codimension of $\Sigma^{I}(f)$.

It is clear by construction that

$$
\mathcal{M}^{m} \supset \Sigma^{i_{1}}(f) \supset \Sigma^{i_{1}, i_{2}}(f) \supset \Sigma^{i_{1}, i_{2}, i_{3}}(f) \supset \cdots
$$

and, if $\Sigma^{i_{1}, \ldots, i_{k}}(f) \neq \emptyset$, then $m \geq i_{1} \geq \cdots \geq i_{k} \geq 0$.

In our developments in Section 3, we will examine how this construction applies to a system of polynomial functions $f: \mathbb{C}^{N} \rightarrow \mathbb{C}^{n}$. In that context, there is no question that the sequence extends indefinitely at any point in $\mathcal{V}(f)$, and that the construction defines an algebraic set at every stage. We examine how this leads to several new concepts in algebraic geometry, the most prominent being the definition of isosingular sets. 


\subsection{Deflation}

Deflation is a method for regularizing nonreduced solution sets. In algebraic geometry, we have the dual notions of ideals (systems of polynomials) and algebraic sets (solutions of the polynomials). Along with these come the dual notions of the radical of an ideal and of reduced algebraic sets. Let $f: \mathbb{C}^{N} \rightarrow \mathbb{C}^{n}$ be a system of polynomials and write $\mathcal{V}(f)=\left\{x \in \mathbb{C}^{N} \mid f(x)=\right.$ $0\}$ for its solution set. An irreducible set $S \in \mathcal{V}(f)$ of dimension $k$ is said to be generically reduced if the Jacobian matrix $J f(x)=\partial f / \partial x$, an $n \times N$ matrix of partial derivatives, has rank $N-k$ when evaluated at general points of $S$. If $S$ is not reduced, it is desirable to replace $f$ with a new system of polynomials for which $S$ is reduced. In the numerical algebraic geometric community, procedures for doing this replacement are called deflation. This is not quite the same as finding the radical ideal $\sqrt{\langle f\rangle}$, because all the solution components of a radical ideal are reduced, while deflation seeks only to reduce one solution component at a time.

Deflation is important in numerical algebraic geometry because Newton's method, an essential tool in the subject, is reliable only for reduced sets. Consider a "square" system $f: \mathbb{C}^{N} \rightarrow \mathbb{C}^{N}$ of polynomial equations that has an isolated solution point. If the Jacobian of $f$ at that point has full rank $N$, Newton's method is a locally quadratically convergent contraction mapping. That is, for all points sufficiently close to the nonsingular isolated solution, repeated application of Newton's method defines a sequence of points which quadratically converge to the solution. In contrast, if the Jacobian of the system has rank less than $N$ at the point, the local behavior of Newton's method can be very different. For example, if $f(x)=x^{2}$, then Newton's method defines a contraction mapping that is only linearly convergent to the origin. A more dramatic example appears in [13, where it is shown that repeated application of Newton's method to the system

$$
f(x, y)=\left[\begin{array}{c}
\frac{29}{16} x^{3}-2 x y \\
y-x^{2}
\end{array}\right]
$$

diverges starting at any point which is not the origin. Clearly, in numerical work, it is a great advantage to work only with nonsingular solution points.

The motivation for deflation is even higher when treating higher dimensional solution components that have generic multiplicity greater than one, because then one encounters a singularity at every point of the component. For example, one may wish to generate other points on a component by tracking a homotopy path defined by the intersection of the component with a moving linear space of complementary dimension. In particular, monodromy loops defined in this fashion are useful in decomposing solution sets into their irreducible components [30, 32, Ch. 15]. If the component has multiplicity greater than one, then the Jacobian of the homotopy function is rank deficient at every point along the monodromy loop, inhibiting efficient numerical computation.

Deflation for an isolated singular solution started with the ideas of Ojika [28, 29. In [21] and [22, these ideas were formalized into an algorithm using symbolic and numerical methods, respectively. Improvements to deflation were made in [10, [23], and [16, §4.1] along with an extension to generically nonreduced irreducible components in [32, §13.3.2, §15.2.2]. Numerical computations can be performed efficiently after this regularization process.

Deflation is applied to a polynomial system at a given solution point to produce an augmented system and an augmented solution point. That is, one step of deflation is formally an operator $\mathcal{D}$ of the form

$$
(g, \widehat{y})=\mathcal{D}(f, \widehat{x}),
$$


where $f: \mathbb{C}^{N} \rightarrow \mathbb{C}^{n}$ is a polynomial system having a solution $\widehat{x} \in \mathcal{V}(f) \subset \mathbb{C}^{N}$ and similarly $g: \mathbb{C}^{M} \rightarrow \mathbb{C}^{m}$ is a polynomial system having a solution $\widehat{y} \in \mathcal{V}(g) \subset \mathbb{C}^{M}$. As we define it below, the output of $\mathcal{D}$ always has $M \geq N, m \geq n$, the first $N$ coordinates of $\widehat{y}$ are $\widehat{x}$, and the first $n$ polynomials in $g$ are $f$. Since the output of deflation is of the same form as the input, we may recurse the operation to generate a series of systems and solution points:

$$
\left(g_{k}, \widehat{y}_{k}\right)=\mathcal{D}^{k}(f, \widehat{x}), \quad k=1,2, \ldots
$$

By convention, we may take $\left(g_{0}, \widehat{y}_{0}\right)=\mathcal{D}^{0}(f, \widehat{x}) \equiv(f, \widehat{x})$.

Several different forms of deflation can be usefully defined. We begin next with a review of one current approach to deflation, which we call "weak deflation" to distinguish it from a stronger form described in Section 4.

\subsection{Weak deflation}

One variant of deflation described in [10] based on [22] is as follows. Let us define

$$
\operatorname{dnull}(f, x)=\operatorname{dim} \text { null } J f(x)
$$

where $J f(x)$ is the Jacobian matrix of $f$ at $x$, and we are speaking of the right null space (the column co-rank). Fix a point $\widehat{x} \in \mathcal{V}(f)$, and let $d=\operatorname{dnull}(f, \widehat{x})$. One step of the deflation process applied to $f$ at $\widehat{x}$ generates a polynomial system $g_{e}: \mathbb{C}^{2 N} \rightarrow \mathbb{C}^{2 n+d}$ and a point $\widehat{y}_{e}=$ $(\widehat{x}, \widehat{\eta}) \in \mathcal{V}\left(g_{e}\right) \subset \mathbb{C}^{2 N}$. The process is to choose a general linear system $\mathcal{L}: \mathbb{C}^{N} \rightarrow \mathbb{C}^{d}$, form the polynomial system $g_{e}$ as

$$
g_{e}(x, \eta)=\left[\begin{array}{c}
f(x) \\
J f(x) \cdot \eta \\
\mathcal{L}(\eta)
\end{array}\right]
$$

and set $\widehat{\eta}$ to be the unique $\eta$ that satisfies $g_{e}(\widehat{x}, \eta)=0$.

Remark 2.1 Deflation could also be constructed using the left null space. For consistency, we use the right null space throughout this article but, in practice, one may use whichever is optimal in terms of the number of polynomials and variables.

In this article, we call (5) weak deflation and denote its operator as $\mathcal{D}_{w, e}$, hence $\left(g_{e}, \widehat{y}_{e}\right)=$ $\mathcal{D}_{w, e}(f, \widehat{x})$. Here, the subscript "e" stands for "extrinsic," which distinguishes it from the intrinsic form, described next.

The intrinsic form of weak deflation, $\mathcal{D}_{w, i}$, replaces the extrinsic slicing linears, $\mathcal{L}$, of equation (5) with an intrinsic form. That is, since $\mathcal{L}$ is a general system of $d$ linear polynomials in $N$ variables, there exists $p \in \mathbb{C}^{N}$ and $A \in \mathbb{C}^{N \times(N-d)}$ such that

$$
\mathcal{V}(\mathcal{L})=\left\{p+A \xi \mid \xi \in \mathbb{C}^{N-d}\right\} .
$$

Denoting the extrinsic and intrinsic deflations as $\left(g_{e}, \widehat{y}_{e}\right)=\mathcal{D}_{w, e}(f, \widehat{x})$ and $\left(g_{i}, \widehat{y}_{i}\right)=\mathcal{D}_{w, i}(f, \widehat{x})$, we define the intrinsic form as

$$
g_{i}(x, \xi)=g_{e}(x, p+A \xi)=\left[\begin{array}{c}
f(x) \\
J f(x) \cdot(p+A \xi)
\end{array}\right],
$$

where we have dropped the equations $\mathcal{L}(p+A \xi)$ because they are identically zero. The output point for intrinsic deflation is $\widehat{y}_{i}=(\widehat{x}, \widehat{\xi})$, where $\widehat{\xi}$ is the unique solution for $\xi$ of $g_{w, i}(\widehat{x}, \xi)=0$. Of 
course, the construction is such that the intrinsic and extrinsic points are related as $\widehat{\eta}=p+A \widehat{\xi}$. As it is clear that $\mathcal{V}\left(g_{w, e}(x, \eta)\right)$ and $\mathcal{V}\left(g_{w, i}(x, \xi)\right)$ are isomorphic, there is no need to distinguish between them, and we may use $\left(g_{w}, \widehat{y}\right)=\mathcal{D}_{w}(f, \widehat{x})$ to mean either of them.

The rationale behind weak deflation is that since the null space of $f$ at $\widehat{x}$ is $d$-dimensional, the intersection of that null space with a generic linear space of complementary dimension isolates a single point, $\widehat{\eta}$, in the null space. This yields a single point $\widehat{y}=(\widehat{x}, \widehat{\eta})$ in the solution set of the augmented system $g$ that projects to $\widehat{x}$ under the natural projection $(x, \eta) \mapsto x$.

The essential property of weak deflation is its effect on multiplicity. Let $\mu(f, \widehat{x})$ denote the multiplicity of an isolated solution point $\widehat{x} \in \mathcal{V}(f)$.

Proposition 2.2 (Deflation of multiplicity) If $\widehat{x}$ is an isolated point in $\mathcal{V}(f)$ having multiplicity $\mu(f, \widehat{x})>1$, then

$$
\mu\left(\mathcal{D}_{w}(f, \widehat{x})\right)<\mu(f, \widehat{x}) .
$$

Proof. See 22 .

Corollary 2.3 (Termination of deflation) If $\widehat{x}$ is an isolated point in $\mathcal{V}(f)$, then the sequence $\mu\left(\mathcal{D}_{w}^{i}(f, \widehat{x})\right)$ for $i=0,1,2, \ldots$ must decrease strictly monotonically until it reaches one, which must happen after at most $\mu(f, \widehat{x})-1$ deflation steps.

The results of [10] show that the number of deflation steps is actually bounded by the depth rather than in terms of the multiplicity.

Both the extrinsic or intrinsic formulations have an associated natural projection. In the extrinsic case, this is $\pi: \mathcal{V}\left(g_{w}\right) \rightarrow \mathcal{V}(f)$ defined by $(x, \eta) \mapsto x$. It is of interest to consider the fibers $\pi^{-1}(x)$ over points $x \in \mathcal{V}(f)$. Clearly, by construction, $\pi^{-1}(\widehat{x})$ is the unique point $\widehat{y}$. Since $\mathcal{L}$ is inhomogeneous, any other points like $\widehat{x}$ that have a $d$-dimensional null space will also have a unique fiber, possibly a point at infinity in the happenstance that the null space is parallel to $\mathcal{V}(\mathcal{L})$. Points with null-space dimension greater than $d$ will have positive dimensional fibers in $\mathcal{V}\left(g_{w}\right)$. The points with null space dimension less than $d$ are of particular interest. If the null space is in general position with respect to $\mathcal{V}(\mathcal{L})$, the fiber will be empty, but there may exist points where the null space meets $\mathcal{V}(\mathcal{L})$. These latter points survive the deflation: they are contained in $\pi\left(\mathcal{V}\left(g_{w}\right)\right)$. We will see an illustration of this in Example 4.5 below.

\section{Deflation sequences}

We describe a new deflation operator, $\mathcal{D}_{\text {det }}$, which uses minors of the Jacobian to enforce a desired condition on the null space dimension of the Jacobian. Let $f: \mathbb{C}^{N} \rightarrow \mathbb{C}^{n}$ be a polynomial system, $\widehat{x} \in \mathcal{V}(f)$, and $d=\operatorname{dnull}(f, \widehat{x})$. Let $\left\{\sigma_{1}, \ldots, \sigma_{m}\right\}$ be the index set of all $(N-d+1) \times(N-$ $d+1$ ) submatrices of an $n \times N$ matrix, which is understood to be empty if $d=\max \{0, N-n\}$. Then $\left(g_{\text {det }}, \widehat{y}_{\text {det }}\right)=\mathcal{D}_{\text {det }}(f, \widehat{x})$ is given by

$$
g_{\operatorname{det}}(x)=\left[\begin{array}{c}
f(x) \\
\operatorname{det} J_{\sigma_{1}} f(x) \\
\vdots \\
\operatorname{det} J_{\sigma_{m}} f(x)
\end{array}\right], \quad \widehat{y}_{\operatorname{det}}=\widehat{x},
$$

where $J_{\sigma} f$ means the submatrix of $J f$ indexed by $\sigma$. We have $m=\left(\begin{array}{c}n \\ N-d+1\end{array}\right) \cdot\left(\begin{array}{c}N \\ N-d+1\end{array}\right)$ and it is clear that $\mathcal{V}(g)=\{x \in \mathcal{V}(f) \mid \operatorname{dnull}(f, x) \geq d\}$. We note the similarities between this description of $\mathcal{V}(g)$ and the definition of $\Sigma^{d}(f)$ presented in (1). 
We have already employed the notation $\mathcal{D}^{k}(f, \widehat{x})$ to mean $k$ successive applications of deflation operator $\mathcal{D}$ to the system $f$ at point $\widehat{x}$. This naturally leads to the definition of a deflation sequence.

Definition 3.1 For a polynomial system $f: \mathbb{C}^{N} \rightarrow \mathbb{C}^{n}$ and a point $\widehat{x} \in \mathcal{V}(f)$, the deflation sequence of $f$ at $\widehat{x}$ is the sequence $\left\{d_{r}(f, \widehat{x})\right\}_{r=0}^{\infty}$ where $d_{k}(f, \widehat{x})=\operatorname{dnull}\left(\mathcal{D}_{\text {det }}^{k}(f, \widehat{x})\right)$.

Remark 3.2 For a deflation step $(g, \widehat{y})=\mathcal{D}_{\operatorname{det}}(f, \widehat{x})$, the only dependence of the polynomial system $g$ on $\widehat{x}$ is the integer $\operatorname{dnull}(f, \widehat{x})$. Letting $\left(g_{k}, \widehat{x}\right)=\mathcal{D}_{\text {det }}^{k}(f, \widehat{x})$, one sees that $g_{k}$ is determined by $f$ and the deflation sequence $d_{j}(f, \widehat{x}), j=0, \ldots, k-1$.

Example 3.3 Consider $f(x, y)=y^{2}-x^{3}, z_{1}=(0,0)$, and $z_{2}=(1,1)$. It is easy to verify that the deflation sequences of $f$ at $z_{1}$ and $z_{2}$, respectively, are

$$
\{2,1,0,0, \ldots\} \text { and }\{1,1,1,1, \ldots\} \text {. }
$$

The following lemma shows that we must also have monotonicity for deflation sequences which is similar to the monotonicity statement in Section 2.1. namely, if $\Sigma^{i_{0}, i_{1}, \ldots, i_{k}}(f)$ is nonempty, then $N \geq i_{0} \geq i_{1} \geq i_{k} \geq 0$.

Lemma 3.4 Let $f: \mathbb{C}^{N} \rightarrow \mathbb{C}^{n}$ be a polynomial system and $\widehat{x} \in \mathcal{V}(f)$ with deflation sequence $\left\{d_{r}(f, \widehat{x})\right\}_{r=0}^{\infty}$. Then, $N \geq d_{k}(f, \widehat{x}) \geq d_{k+1}(f, \widehat{x}) \geq 0$ for every $k \geq 0$.

Proof. Clearly, $N \geq d_{k}(f, \widehat{x}) \geq 0$ for all $k \geq 0$. Due to the recursive nature of the definition of $d_{k}(f, \widehat{x})$, it suffices to show that $d_{0}(f, \widehat{x}) \geq d_{1}(f, \widehat{x})$. This is equivalent to showing that $\operatorname{dnull}(f, \widehat{x}) \geq \operatorname{dnull}\left(g_{\text {det }}, \widehat{x}\right)$ where $g_{\text {det }}$ is defined as in $(7)$ which follows immediately from the definition of $g_{\text {det }}$.

Since deflation sequences are monotonically decreasing sequences of nonnegative integers, they must stabilize. That is, there exists $k^{*} \geq 0$ and $d \geq 0$ such that

$$
d_{0}(f, \widehat{x}) \geq d_{1}(f, \widehat{x}) \geq \cdots \geq d_{k^{*}-1}(f, \widehat{x})>d_{k^{*}}(f, \widehat{x})=d_{k^{*}+1}(f, \widehat{x})=\cdots=d .
$$

Deflation is said to terminate if $d=0$. In this case, deflation has restored quadratic convergence of Newton's method since the corresponding root of the deflated system is nonsingular.

\subsection{Deflation sequences for irreducible algebraic sets}

Deflation sequences can be naturally extended to irreducible algebraic sets. In particular, if $V \subset \mathcal{V}(f)$ is a nonempty irreducible algebraic set, there is a nonempty Zariski open subset $U \subset V$ such that $\left\{d_{r}(f, x)\right\}_{r=0}^{\infty}=\left\{d_{r}(f, y)\right\}_{r=0}^{\infty}$ for any $x, y \in U$. Throughout this section, we say that the points in $U$ are the general points of $V$. We formally define the deflation sequence for $V$ based on $U$.

Definition 3.5 For a polynomial system $f: \mathbb{C}^{N} \rightarrow \mathbb{C}^{n}$ and a nonempty irreducible algebraic set $V \subset \mathcal{V}(f)$, the deflation sequence of $f$ at $V$, denoted $\left\{d_{r}(f, V)\right\}_{r=0}^{\infty}$, is the deflation sequence of $f$ at any generic point $x^{*} \in V$, that is, $d_{k}(f, V)=\operatorname{dnull}\left(\mathcal{D}_{\text {det }}^{k}\left(f, x^{*}\right)\right)$.

The polynomial system $f$ and the deflation sequence $\left\{d_{r}(f, V)\right\}_{r=0}^{\infty}$ define the sequence of polynomial systems $g_{k}$ given by $\left(g_{k}, x^{*}\right)=\mathcal{D}_{\text {det }}^{k}\left(f, x^{*}\right)$ for generic $x^{*} \in V$. As discussed in 
Remark 3.2 the polynomial systems $g_{k}$ only depend upon the deflation sequence $d_{j}\left(f, x^{*}\right)=$ $d_{j}(f, V), j=0,1, \ldots, k-1$. Moreover, we must have $V \subset \mathcal{V}\left(g_{k}\right)$ for each $k \geq 0$.

Suppose that $x \in V$ such that $\left\{d_{r}(f, x)\right\}_{r=0}^{\infty} \neq\left\{d_{r}(f, V)\right\}_{r=0}^{\infty}$. Then, there must be a first element in the sequence where they differ: say $d_{k}(f, x)=d_{k}(f, V)$ for $0 \leq k<\ell$ but $d_{\ell}(f, x) \neq d_{\ell}(f, V)$. By genericity and upper semi-continuity, we must have

$$
d_{\ell}(f, x)>d_{\ell}(f, V) .
$$

The recursive nature of deflation yields that $\mathcal{D}^{j}\left(\mathcal{D}^{k}(f, x)\right)=\mathcal{D}^{j+k}(f, x)$. Since

$$
\mathcal{D}^{j}\left(g_{k}, x\right)=\mathcal{D}^{j}\left(\mathcal{D}^{k}(f, x)\right)=\mathcal{D}^{j+k}(f, x)
$$

it follows that the deflation sequences of $V$ with respect to $f$ and $g_{k}$ are naturally related in that

$$
d_{j+k}(f, V)=d_{j}\left(g_{k}, V\right) .
$$

The following lemma shows that each term in the deflation sequence is an upper bound on the dimension.

Lemma 3.6 If $f: \mathbb{C}^{N} \rightarrow \mathbb{C}^{n}$ is a polynomial system and $V \subset \mathcal{V}(f)$ is a nonempty irreducible algebraic set, then, for every $k \geq 0$,

$$
d_{k}(f, V) \geq \operatorname{dim} V .
$$

In particular, $\lim _{k \rightarrow \infty} d_{k}(f, V) \geq \operatorname{dim} V$.

Proof. Clearly, for every $k \geq 0$, we have $d_{k}(f, V)=d_{0}\left(g_{k}, V\right) \geq \operatorname{dim} V$.

Since $V \subset \mathcal{V}\left(g_{k}\right)$ is an irreducible algebraic set, there exists some irreducible component $Z \subset \mathcal{V}\left(g_{k}\right)$ such that $V \subset Z$. The following lemma relates the deflation sequence for $V$ and $Z$ with respect to $f$.

Lemma 3.7 Suppose that $f: \mathbb{C}^{N} \rightarrow \mathbb{C}^{n}$ is a polynomial system, $V \subset \mathcal{V}(f)$ is a nonempty irreducible algebraic set, $\widehat{x} \in V$ is general, and $k \geq 1$. Let $\left(g_{k}, \widehat{x}\right)=\mathcal{D}_{\text {det }}^{k}(f, \widehat{x})$. Then, for every irreducible component $Z \subset \mathcal{V}\left(g_{k}\right)$ which contains $V$,

$$
d_{j}(f, V)=d_{j}(f, Z) \text { for } j=0,1, \ldots, k-1 .
$$

Proof. Let $\widehat{z} \in Z$ be general.

Since $\widehat{x} \in V \subset Z$, we must have $d_{0}(f, \widehat{x}) \geq d_{0}(f, \widehat{z})$. Since $\mathcal{D}_{\text {det }}$ removes points having null space dimension less than that of the input point, we know $d_{0}(f, \widehat{x})=d_{0}(f, \widehat{z})$.

The proof is now complete for the case $k=1$, so we assume that $k \geq 2$ and proceed recursively. Suppose that $\ell \in\{0,1, \ldots, k-2\}$ such that $d_{j}(f, \widehat{x})=d_{j}(f, \widehat{z})$ for $j=0,1, \ldots, \ell$. Since $\widehat{x}, \widehat{z} \in Z$ and $\widehat{z} \in Z$ is general, we know $d_{\ell+1}(f, \widehat{x}) \geq d_{\ell+1}(f, \widehat{z})$. Since $\ell+1<k$ and $\mathcal{D}_{\text {det }}$ removes points having null space dimension less than that of the input point, we know $d_{\ell+1}(f, \widehat{x})=d_{\ell+1}(f, \widehat{z})$.

This recursive program yields $d_{j}(f, \widehat{x})=d_{j}(f, \widehat{z})$ for $j=0,1, \ldots, k-1$. 


\section{Strong deflation}

By modifying the weak deflation operators $\mathcal{D}_{w, i}$ and $\mathcal{D}_{w, e}$ defined in Section 2.3 with results from [2], we obtain strong deflation operators $\mathcal{D}_{s, i}$ and $\mathcal{D}_{s, e}$. We show these strong deflation operators are equivalent, in a sense that we will make precise, to the determinantal deflation operator $\mathcal{D}_{\text {det }}$ defined in Section 3.

Our motivation for introducing equivalent forms of the deflation $\mathcal{D}_{\text {det }}$ is twofold, namely, the number of polynomials in $g_{\text {det }}$ defined in $(7)$ can be quite large and determinants often produce polynomials of high degree. In numerical work, it can be beneficial to employ a different formulation of deflation that introduces additional variables but reduces the number of polynomials and does not increase degrees. In [2, a method is given for specifying the condition $\operatorname{dim}$ null $Q \geq d$ for a matrix $Q \in \mathbb{C}^{n \times N}$. The idea is that a full-rank matrix $B \in \mathbb{C}^{N \times N}$ generates a generic patch on the Grassmannian, $G r(d, N)$, of $d$-dimensional homogeneous linear spaces in $\mathbb{C}^{N}$ parameterized by $\Xi \in \mathbb{C}^{(N-d) \times d}$ such that

$$
G(B, \Xi)=B \cdot\left[\begin{array}{l}
I \\
\Xi
\end{array}\right]
$$

is a basis for the $d$-dimensional subspace. In other words, each choice of $\Xi$ gives $d$ linearly independent columns in $G(B, \Xi)$ and conversely almost all points in $\operatorname{Gr}(d, N)$ have a corresponding unique $\Xi$ such that $G(B, \Xi)$ generates the same subspace. There is a hypersurface in $\operatorname{Gr}(d, N)$ of points that have no corresponding $\Xi$, but for any point in $\operatorname{Gr}(d, N)$, there is a Zariski-open subset $U \subset \mathbb{C}^{N \times N}$ such that $B \in U$ gives a corresponding representative $\Xi$. Accordingly, if $Q \in \mathbb{C}^{n \times N}$ has $\operatorname{dim}$ null $Q=d$, then for generic $B$,

$$
Q \cdot B \cdot\left[\begin{array}{c}
I \\
\Xi
\end{array}\right]=0
$$

has a unique solution for $\Xi$.

Using a patch on $\operatorname{Gr}(d, N)$, an intrinsic formulation of strong deflation, $\left(g_{i}, \widehat{y}_{i}\right)=\mathcal{D}_{s, i}(f, \widehat{x})$ gives $g_{i}: \mathbb{C}^{N+(N-d) d} \rightarrow \mathbb{C}^{n+n d}$ as

$$
g_{i}(x, \Xi)=\left[\begin{array}{c}
f(x) \\
J f(x) \cdot B \cdot\left[\begin{array}{l}
I \\
\Xi
\end{array}\right]
\end{array}\right]
$$

where $B \in \mathbb{C}^{N \times N}$ is chosen at random. The corresponding $\widehat{y}_{i}$ is given as $(\widehat{x}, \widehat{\Xi})$, where $\widehat{\Xi}$ is the unique solution for $\Xi$ of $g_{i}(\widehat{x}, \Xi)=0$. (Due to the argument in the previous paragraph, this solution exists and is unique for any $B$ in a Zariski-open subset of $\mathbb{C}^{N \times N}$.)

Remark 4.1 As discussed in Remark 2.1, we could also perform this construction using the left null space.

This version of strong deflation is closely related to the weak deflations presented earlier. Let $P \in \mathbb{C}^{N \times d}$ and $A \in \mathbb{C}^{N \times(N-d)}$ be submatrices such that $B=[P A]$. Denote the columns of $P$ as $p_{1}, \ldots, p_{d}$ and the columns of $\Xi$ as $\xi_{1}, \ldots, \xi_{d}$. Then $g_{i}(x, \Xi)$ in equation 10 becomes

$$
g_{i}\left(x, \xi_{1}, \ldots, \xi_{d}\right)=\left[\begin{array}{c}
f(x) \\
J f(x) \cdot\left(p_{1}+A \xi_{1}\right) \\
\vdots \\
J f(x) \cdot\left(p_{d}+A \xi_{d}\right)
\end{array}\right] .
$$


This is similar to the intrinsic deflation of equation (6) differing only in an increase from 1 to $d$ in the number of appended subsystems. One can convert this to an equivalent extrinsic form by introducing linear systems $\mathcal{L}_{i}: \mathbb{C}^{N} \rightarrow \mathbb{C}^{d}, i=1, \ldots, d$, such that $\mathcal{V}\left(\mathcal{L}_{i}\right)=\left\{p_{i}+A \xi \mid \xi \in \mathbb{C}^{N-d}\right\}$. In particular, these are of the form

$$
\mathcal{L}_{i}\left(\eta_{i}\right)=A^{\perp}\left(\eta_{i}-p_{i}\right),
$$

where $A^{\perp}$ is a $d \times N$ basis for the left null space of $A$. (That is, rank $A^{\perp}=d$ and $A^{\perp} A=0$.) With these systems we write an extrinsic system whose solution set is isomorphic to $\mathcal{V}\left(g_{i}(x, \xi)\right)$ as

$$
g_{e}\left(x, \eta_{1}, \ldots, \eta_{d}\right)=\left[\begin{array}{c}
f(x) \\
J f(x) \cdot \eta_{1} \\
\mathcal{L}_{1}\left(\eta_{1}\right) \\
\vdots \\
J f(x) \cdot \eta_{d} \\
\mathcal{L}_{d}\left(\eta_{d}\right)
\end{array}\right]
$$

where $g_{e}: \mathbb{C}^{(d+1) N} \rightarrow \mathbb{C}^{(d+1) n+d^{2}}$. Accordingly, the extrinsic form of strong deflation is $\left(g_{e}, \widehat{y}_{e}\right)=$ $\mathcal{D}_{s, e}(f, \widehat{x})$, where $\widehat{y}_{e}=\left\{\widehat{x}, \widehat{\eta}_{1}, \ldots, \widehat{\eta}_{d}\right\}$ with $\widehat{\eta}_{i}=p_{i}+A \widehat{\xi}_{i}, i=1, \ldots, d$.

Proposition 4.2 (Equivalence of strong deflations) The deflations $\mathcal{D}_{s, \mathrm{det}}, \mathcal{D}_{s, e}$, and $\mathcal{D}_{s, i}$ are all equivalent in the sense that:

1. $\mathcal{V}\left(\mathcal{D}_{\operatorname{det}}(f, \widehat{x})\right), \mathcal{V}\left(\mathcal{D}_{s, e}(f, \widehat{x})\right)$, and $\mathcal{V}\left(\mathcal{D}_{s, i}(f, \widehat{x})\right)$ are all generically isomorphic, and

2. $\operatorname{dnull}\left(\mathcal{D}_{\operatorname{det}}(f, \widehat{x})\right)=\operatorname{dnull}\left(\mathcal{D}_{s, e}(f, \widehat{x})\right)=\operatorname{dnull}\left(\mathcal{D}_{s, i}(f, \widehat{x})\right)$.

Proof. The equivalence of $\mathcal{D}_{s, e}$ and $\mathcal{D}_{s, i}$ is clear from their construction. The equivalence of $\mathcal{D}_{\text {det }}$ to these is proven as follows. Let $\left(g_{\text {det }}, \widehat{x}\right)=\mathcal{D}_{\operatorname{det}}(f, \widehat{x}),\left(g_{e},(\widehat{x}, \widehat{\eta})\right)=\mathcal{D}_{s, e}(f, \widehat{x})$, and $d=\operatorname{dnull}(f, \widehat{x})$. Let $I$ be the ideal generated by $g_{\text {det }}$, and let $J$ be the ideal generated by $g_{e}$. We know that $I=J \cap C[x]$ and locally near $\widehat{x}$, the projection map from $\mathcal{V}(J)$ to $\mathcal{V}(I)$ is an isomorphism. In particular, we can find a ball $B$ containing $\widehat{x}$ such that every point in $B \cap \mathcal{V}(f)$ has a null space of $J f$ of dimension at most $d$. Hence, every point in $B \cap \mathcal{V}(I)$ has a null space of $J f$ of dimension exactly $d$. This implies that locally near $\widehat{x}$ in $\mathcal{V}(I)$, the extra variables $\widehat{\eta}$ are described by rational functions of $x$. Therefore, the dimension of the tangent space of the ideal $J$ at $(\widehat{x}, \widehat{\eta})$ is the same as the dimension of the tangent space of the ideal $I$ at $\widehat{x}$.

Due to their equivalence, we need not distinguish between the three forms of strong deflation: in the following proposition, we refer to them all as simply $\mathcal{D}_{s}$.

Proposition 4.3 Proposition 2.2 and Corollary 2.3, which are stated for weak deflation $\mathcal{D}_{w}$, also hold for strong deflation, $\mathcal{D}_{s}$.

Proof. Let $d=\operatorname{dnull}(f, \widehat{x})$. The statement is trivial if $d=0$. If $d=1$, then strong and weak deflation are equivalent. If $d>1$, then comparing equations (5) and $\sqrt{13}$, one sees that the ideal for a step of strong deflation is a sum of $d$ ideals, each being the ideal for a step of weak deflation. In particular, the multiplicity after one step of strong deflation is bounded above by the multiplicity after one step of weak deflation.

For the same reason, the depth bounded results of [10] hold for strong deflation. 
Remark 4.4 Suppose that $\widehat{x}$ is an isolated root of $f$ of multiplicity $m$ and depth $\delta$ with deflation sequence $\left\{d_{r}(f, \widehat{x})\right\}_{r=0}^{\infty}$ which stabilizes at $k^{*} \geq 0$ and limits to $d$. That is, either $k^{*}=0$ with $d_{k}(f, \widehat{x})=d$ for all $k \geq 0$ or $k^{*}>0$ with $d_{k^{*}-1}(f, \widehat{x})>d_{k^{*}}(f, \widehat{x})=d_{k^{*}+1}(f, \widehat{x})=\cdots=d$. Interpreting the results of [10, 22] in this context yield that $k^{*} \leq \delta \leq m-1$ and $d=0$.

The advantage of strong over weak deflation is that it builds a complete basis for the null space of $J f(x)$. That is, given $\widehat{x} \in \mathcal{V}(f)$ with $\operatorname{dnull}(f, \widehat{x})=d$, then, for strong extrinsic deflation, with probability one the solution of $g_{s, e}\left(\widehat{x}, \eta_{1}, \ldots, \eta_{d}\right)=0$ for $\left(\eta_{1}, \ldots, \eta_{d}\right)$ yields a set of $d$ linearly independent vectors in null $J f(\widehat{x})$. (To be more precise, linear independence holds for $B$ in a dense Zariski-open subset of $\mathbb{C}^{N \times N}$.) In contrast, for weak extrinsic deflation, the solution of $g_{w, e}(\widehat{x}, \eta)=0$ yields only one vector in the null space. As claimed in the proof of Proposition 4.2 . the linear independence of $\eta_{1}, \ldots, \eta_{d}$ extends to an open ball around $\widehat{x}$, so $\mathcal{V}\left(g_{s}\right)$ has a component containing $\left(\widehat{x}, \eta_{1}, \ldots, \eta_{d}\right)$ with dim null $J f(x) \geq d$, a stronger condition than one obtains using $g_{w, e}$. For $d>1$, the drawback is that $g_{s, e}$ has more variables and polynomials than $g_{w, e}$. In this article, we are focused on performing deflation in situations where the extra conditions imposed by strong deflation play to our advantage. One such case is illustrated by the following example.

Example 4.5 Consider $f\left(x_{1}, x_{2}, x_{3}\right)=x_{1}^{2}+x_{2}^{2}+x_{3}^{2}$ and $\widehat{x}=(0,0,0)$. Clearly, $N=3, n=1$, and $d=\operatorname{dnull}(f, \widehat{x})=3$. Because $d=N$, the symbols $A$ and $\xi$ disappear, and we have

$$
g_{w, i}\left(x_{1}, x_{2}, x_{3}\right)=\left[\begin{array}{c}
x_{1}^{2}+x_{2}^{2}+x_{3}^{2} \\
2\left(x_{1} p_{11}+x_{2} p_{21}+x_{3} p_{31}\right)
\end{array}\right]
$$

and

$$
g_{s, i}\left(x_{1}, x_{2}, x_{3}\right)=\left[\begin{array}{c}
x_{1}^{2}+x_{2}^{2}+x_{3}^{2} \\
2\left(x_{1} p_{11}+x_{2} p_{21}+x_{3} p_{31}\right) \\
2\left(x_{1} p_{12}+x_{2} p_{22}+x_{3} p_{32}\right) \\
2\left(x_{1} p_{13}+x_{2} p_{23}+x_{3} p_{33}\right)
\end{array}\right],
$$

where, for $1 \leq i, j \leq 3, p_{i j} \in \mathbb{C}$ are random. Since $P=\left(p_{i j}\right)$ is general, one sees that strong deflation isolates $\widehat{x}$, i.e., $\mathcal{V}\left(g_{s, i}\right)=\{\widehat{x}\}$,

and $J g_{s, i}(\widehat{x})$ is full rank. On the other hand, for weak deflation, $\mathcal{V}\left(g_{w, i}\right)$ is a curve. This is because $g_{s, i}$ picks out points in the neighborhood of $\widehat{x}$ that have a 3 -dimensional null space, which only leaves $\widehat{x}$, while $g_{w, i}$ only imposes the condition that locally dim null $J f(x) \geq 2$. One can verify that one more weak deflation step isolates the origin while a third weak deflation step is required to construct a polynomial system for which $\widehat{x}$ corresponds to a nonsingular isolated solution.

Simplification of notation: From this point forward, we only consider strong deflation, and in light of Proposition 4.2, for most purposes we do not need to distinguish between different forms of strong deflation. Accordingly, from here on, let $\mathcal{D}$ mean any of $\mathcal{D}_{\text {det }}, \mathcal{D}_{s, e}$, or $\mathcal{D}_{s, i}$. Moreover, each of the deflations has a natural projection that sends $y$ in $(g, y)=\mathcal{D}(f, x)$ back to $x: \pi(y)=x$. We use the symbol $\pi$ for all of these, regardless of which underlying formulation is used. Similarly, when successive deflations are involved, we use $\pi_{k}$ to denote the natural projection that sends $y_{k}$ in $\left(g_{k}, y_{k}\right)=\mathcal{D}^{k}(f, x)$ back to $x$. On occasions where we need to write explicit formulae, we may revert to the more specific notations.

\subsection{Strong deflation and irreducible components}

Let $f: \mathbb{C}^{N} \rightarrow \mathbb{C}^{n}$ be a polynomial system and $V \subset \mathcal{V}(f)$ be a nonempty irreducible algebraic set. As discussed in Section 3.1. the deflation sequence of $V$ with respect to $f$ is the deflation sequence 
of any general point in $V$, say $x^{*}$, with respect to $f$. Lemma 3.6 shows that $\lim _{k \rightarrow \infty} d_{k}(f, V) \geq$ $\operatorname{dim} V$. We will show that if $V$ is an irreducible component of $\mathcal{V}(f)$, then this is an equality. This can be considered as the interpretation of the deflation results of [32, §13.3.2, §15.2.2] applied to irreducible components using this context.

For each $k \geq 0$, let $\left(g_{k}, y_{k}\right)=\mathcal{D}^{k}\left(f, x^{*}\right)$. If $x \in V$ such that $x$ and $V$ have the same deflation sequence, then, for each $k \geq 0$, there exists a unique $y \in \mathcal{V}\left(g_{k}\right)$ such that $\pi_{k}(y)=x$. Define

$$
W_{k}=\overline{\left\{y \in \mathcal{V}\left(g_{k}\right) \mid \pi_{k}(y) \in V \text { with }\left\{d_{r}\left(f, \pi_{k}(y)\right)\right\}_{r=0}^{\infty}=\left\{d_{r}(f, V)\right\}_{r=0}^{\infty}\right\}} \subset \mathcal{V}\left(g_{k}\right) .
$$

Hence, $V$ is generically isomorphic to $W_{k}$ and, in particular, $W_{k}$ is an irreducible algebraic set with $\operatorname{dim} W_{k}=\operatorname{dim} V$. We summarize this in the following lemma.

Lemma 4.6 If $f: \mathbb{C}^{N} \rightarrow \mathbb{C}^{n}$ is a polynomial system and $V \subset \mathcal{V}(f)$ is a nonempty irreducible algebraic set, then, for every $k \geq 0$, the set $W_{k}$ defined by (14) is an irreducible algebraic set that is generically isomorphic to $V$. In particular, $\operatorname{dim} W_{k}=\operatorname{dim} V$.

If $g: \mathbb{C}^{N} \rightarrow \mathbb{C}^{n}$ is a polynomial system and $V \subset \mathcal{V}(g)$ is an irreducible component, then define $\mu(g, V)$ to be the multiplicity of $V$ with respect to $g$. In particular, if $d=\operatorname{dim} V$ and $\mathcal{L}: \mathbb{C}^{N} \rightarrow \mathbb{C}^{d}$ are general, then $\mu(f, V)$ is equal to $\mu(\{f, \mathcal{L}\}, x)$ for any of the finitely many points $x \in V \cap \mathcal{V}(\mathcal{L})$.

The following lemma shows that the limit of the deflation sequence for an irreducible component is its dimension.

Lemma 4.7 If $f: \mathbb{C}^{N} \rightarrow \mathbb{C}^{n}$ is a polynomial system and $V \subset \mathcal{V}(f)$ is a nonempty irreducible component, then $\lim _{k \rightarrow \infty} d_{k}(f, V)=\operatorname{dim} V$.

Proof. Since the statement is trivial when $d_{0}(f, V)=\operatorname{dim} V$, we will assume that $d_{0}(f, V)>$ $\operatorname{dim} V$. In particular, we know that $\mu(f, V)>1$. Additionally, for the case $\operatorname{dim} V=0$, the statement follows from Proposition 4.3. so only the case $\operatorname{dim} V>0$ requires further consideration.

We first note a relationship between weak deflation and slicing. Let $\ell=\operatorname{dim} V, d=$ $d_{0}(f, V)>\ell$, and $\mathcal{M}: \mathbb{C}^{N} \rightarrow \mathbb{C}^{\ell}$ be a system of general linear polynomials. Let $A \in \mathbb{C}^{\ell \times N}$ and $a \in \mathbb{C}^{\ell}$ such that $\mathcal{M}(x)=A x+a$.

Fix $\widehat{x} \in V \cap \mathcal{V}(\mathcal{M})$ and consider $\left(g_{1}, \widehat{y}_{1}\right)=\mathcal{D}_{w}(f, \widehat{x})$ and $\left(g_{2}, \widehat{y}_{2}\right)=\mathcal{D}_{w}(\{f, \mathcal{M}\}, \widehat{x})$. Using an extrinsic formulation of weak deflation as in (5), we can write

$$
g_{1}(x, \eta)=\left[\begin{array}{c}
f(x) \\
J f(x) \cdot \eta \\
\mathcal{L}_{1}(\eta)
\end{array}\right] \text { and } g_{2}(x, \xi)=\left[\begin{array}{c}
f(x) \\
\mathcal{M}(x) \\
J f(x) \cdot \xi \\
A \cdot \xi \\
\mathcal{L}_{2}(\xi)
\end{array}\right]
$$

where $\mathcal{L}_{1}: \mathbb{C}^{N} \rightarrow \mathbb{C}^{d}$ and $\mathcal{L}_{2}: \mathbb{C}^{N} \rightarrow \mathbb{C}^{d-\ell}$ are systems of general linear polynomials. Since $d>\ell$, by using Gaussian elimination, without loss of generality, we can assume that

$$
\mathcal{L}_{1}(\eta)=\left[\begin{array}{c}
B \cdot \eta \\
C \cdot \eta+c
\end{array}\right] \text { and } \mathcal{L}_{2}(\xi)=C \cdot \xi+c
$$


where $B \in \mathbb{C}^{\ell \times N}, C \in \mathbb{C}^{(d-\ell) \times N}$, and $c \in \mathbb{C}^{(d-\ell) \times N}$ are general. That is,

$$
\left[\begin{array}{c}
g_{1}(x, \eta) \\
\mathcal{M}(x)
\end{array}\right]=\left[\begin{array}{c}
f(x) \\
A x+a \\
J f(x) \cdot \eta \\
B \cdot \eta \\
C \cdot \eta+c
\end{array}\right] \text { and } g_{2}(x, \xi)=\left[\begin{array}{c}
f(x) \\
A x+a \\
J f(x) \cdot \xi \\
A \cdot \xi \\
C \cdot \xi+c
\end{array}\right]
$$

Since $\widehat{x}$ is isolated in $\mathcal{V}(f, \mathcal{M})$ with $\mu(\{f, \mathcal{M}\}, \widehat{x})=\mu(f, V)>1$, Proposition 2.2 yields that $\mu\left(g_{2}, \widehat{y}_{2}\right)<\mu(\{f, \mathcal{M}\}, \widehat{x})$. Since $B \in \mathbb{C}^{\ell \times N}$ is general, comparing the polynomial systems in 15 clearly yields $\mu\left(\left\{g_{1}, \mathcal{M}\right\}, \widehat{y}_{1}\right) \leq \mu\left(g_{2}, \widehat{y}_{2}\right)<\mu(f, V)$.

Let $\left(g_{s}, \widehat{y}_{s}\right)=\mathcal{D}_{s}(f, \widehat{x})$ where we use the extrinsic formulation of strong deflation. Comparing $\left[\begin{array}{c}g_{s}\left(x, \eta_{1}, \ldots, \eta_{d}\right) \\ \mathcal{M}(x)\end{array}\right]$ with $\left[\begin{array}{c}g_{1}(x, \eta) \\ \mathcal{M}(x)\end{array}\right]$ using a similar argument to that in the proof of Proposition 4.3 , we have that

$$
\mu\left(\left\{g_{s}, \mathcal{M}\right\}, \widehat{y}_{s}\right) \leq \mu\left(\left\{g_{1}, \mathcal{M}\right\}, \widehat{y}_{1}\right)<\mu(f, V) .
$$

In particular, after at most $\mu(f, V)-1$ deflation steps, the deflation sequence $d_{k}(f, V)$ must stabilize to $\operatorname{dim} V$.

\section{$5 \quad$ Isosingular sets}

For a polynomial system $f$ and a nonempty irreducible algebraic set $V \subset \mathcal{V}(f)$, Lemma 3.6 shows that $\lim _{k \rightarrow \infty} d_{k}(f, V) \geq \operatorname{dim} V$ with Lemma 4.7 showing that, when $V$ is an irreducible component, this is actually an equality. We will define isosingular sets as the closures of sets of points with the same deflation sequence and show these sets are exactly those for which this inequality is actually an equality.

For any sequence $\left\{\delta_{r}\right\}_{r=0}^{\infty}$ of nonnegative integers, define

$$
X_{f}^{\infty}\left(\left\{\delta_{r}\right\}_{r=0}^{\infty}\right)=\overline{\left\{x \in \mathcal{V}(f) \mid d_{k}(f, x)=\delta_{k} \text { for all } k \geq 0\right\}} \subset \mathcal{V}(f)
$$

and, for each $j \geq 0$, define

$$
X_{f}^{j}\left(\left\{\delta_{r}\right\}_{r=0}^{\infty}\right)=\overline{\left\{x \in \mathcal{V}(f) \mid d_{k}(f, x)=\delta_{k} \text { for } 0 \leq k \leq j\right\}} \subset \mathcal{V}(f) .
$$

Clearly, each $X_{f}^{j}\left(\left\{\delta_{r}\right\}_{r=0}^{\infty}\right)$ is an algebraic set with $X_{f}^{j+1}\left(\left\{\delta_{r}\right\}_{r=0}^{\infty}\right) \subset X_{f}^{j}\left(\left\{\delta_{r}\right\}_{r=0}^{\infty}\right)$ and

$$
X_{f}^{\infty}\left(\left\{\delta_{r}\right\}_{r=0}^{\infty}\right)=\bigcap_{j=0}^{\infty} X_{f}^{j}\left(\left\{\delta_{r}\right\}_{r=0}^{\infty}\right) .
$$

By the descending chain condition on algebraic sets, there exists $\ell \geq 0$ such that, for all $j \geq \ell$,

$$
X_{f}^{j}\left(\left\{\delta_{r}\right\}_{r=0}^{\infty}\right)=X_{f}^{\ell}\left(\left\{\delta_{r}\right\}_{r=0}^{\infty}\right) .
$$

This yields $X_{f}^{\infty}\left(\left\{\delta_{r}\right\}_{r=0}^{\infty}\right)=X_{f}^{\ell}\left(\left\{\delta_{r}\right\}_{r=0}^{\infty}\right)$. In particular, $X_{f}^{\infty}\left(\left\{\delta_{r}\right\}_{r=0}^{\infty}\right)$ is an algebraic set and we call each of its nonempty irreducible components an isosingular set. 
Definition 5.1 If $f: \mathbb{C}^{N} \rightarrow \mathbb{C}^{n}$ is a polynomial system and $V \subset \mathcal{V}(f)$ is a nonempty irreducible algebraic set, then $V$ is called an isosingular set with respect to $f$ if $V$ is an irreducible component of $X_{f}^{\infty}\left(\left\{d_{r}(f, V)\right\}_{r=0}^{\infty}\right)$.

A point $x \in \mathcal{V}(f)$ is an isosingular point with respect to $f$ if $\{x\}$ is an isosingular set with respect to $f$.

Theorem 5.2 If $f: \mathbb{C}^{N} \rightarrow \mathbb{C}^{n}$ is a polynomial system and $V \subset \mathcal{V}(f)$ is an irreducible component, then $V$ is an isosingular set with respect to $f$.

Proof. The statement follows immediately from the fact that $V \subset X_{f}^{\infty}\left(\left\{d_{r}(f, V)\right\}_{r=0}^{\infty}\right) \subset \mathcal{V}(f)$.

The following lemma shows that isosingular sets correspond to the closure of the natural projection of irreducible components of deflations.

Lemma 5.3 If $f: \mathbb{C}^{N} \rightarrow \mathbb{C}^{n}$ is a polynomial system, $V \subset \mathcal{V}(f)$ is a nonempty irreducible algebraic set, and $\widehat{x} \in V$ such that $d_{k}(f, \widehat{x})=d_{k}(f, V)$ for all $k \geq 0$, then the following are equivalent.

1. $V$ is an isosingular set with respect to $f$.

2. There exists $k \geq 0$ such that if $\left(g_{k}, \widehat{y}_{k}\right)=\mathcal{D}^{k}(f, \widehat{x})$ and $W_{k}$ is defined by (14), then $W_{k}$ is an irreducible component of $\mathcal{V}\left(g_{k}\right)$ with $V=\overline{\pi_{k}\left(W_{k}\right)}$.

Proof. For each $\ell \geq 0$, since, by Lemma 4.6. $W_{\ell}$ is an irreducible algebraic subset of $\mathcal{V}\left(g_{\ell}\right)$, there exists an irreducible component of $\mathcal{V}\left(g_{\ell}\right)$ which contains $W_{\ell}$. Let $Z_{\ell}$ be the union of such irreducible components of $\mathcal{V}\left(g_{\ell}\right)$. In particular, there exists $m_{\ell} \geq 1$ and irreducible components $Z_{\ell, 1}, \ldots, Z_{\ell, m_{\ell}} \subset \mathcal{V}\left(g_{\ell}\right)$ such that

$$
Z_{\ell}=\bigcup_{i=1}^{m_{\ell}} Z_{\ell, i}
$$

Lemma 3.7 yields, for each $\ell \geq 1$ and $j=1, \ldots, m_{\ell}$,

$$
V \subset \overline{\pi_{\ell}\left(Z_{\ell, j}\right)} \subset X_{f}^{\ell-1}\left(\left\{d_{r}(f, V)\right\}_{r=0}^{\infty}\right) .
$$

Additionally, it is clear that we must have

$$
V \subset \overline{\pi_{\ell+1}\left(Z_{\ell+1}\right)} \subset \overline{\pi_{\ell}\left(Z_{\ell}\right)}
$$

By the descending chain condition, there exists $k \geq 0$ such that, for all $\ell \geq k+1$,

$$
\overline{\pi_{k}\left(Z_{k}\right)}=\overline{\pi_{\ell}\left(Z_{\ell}\right)} \subset X_{f}^{\ell-1}\left(\left\{d_{r}(f, V)\right\}_{r=0}^{\infty}\right) .
$$

Thus, we know that

$$
V \subset \overline{\pi_{k}\left(Z_{k}\right)} \subset X_{f}^{\infty}\left(\left\{d_{r}(f, V)\right\}_{r=0}^{\infty}\right) .
$$

Suppose that $V$ is an isosingular set with respect to $f$, that is, $V$ is an irreducible component of $X_{f}^{\infty}\left(\left\{d_{r}(f, V)\right\}_{r=0}^{\infty}\right)$. In particular, we know that

$$
V \subset \overline{\pi_{k}\left(Z_{k, 1}\right)} \subset \overline{\pi_{k}\left(Z_{k}\right)} \subset X_{f}^{\infty}\left(\left\{d_{r}(f, V)\right\}_{r=0}^{\infty}\right) .
$$


Since $\overline{\pi_{k}\left(Z_{k, 1}\right)}$ is an irreducible algebraic subset of $X_{f}^{\infty}\left(\left\{d_{r}(f, V)\right\}_{r=0}^{\infty}\right)$ which contains the irreducible component $V$ of $X_{f}^{\infty}\left(\left\{d_{r}(f, V)\right\}_{r=0}^{\infty}\right)$, it follows that $V=\overline{\pi_{k}\left(Z_{k, 1}\right)}=\overline{\pi_{k}\left(W_{k}\right)}$. Since $\pi_{k}$ restricted to $\pi_{k}^{-1}(V)$ is generically an isomorphism, we know that $Z_{k, 1}=W_{k}$ yielding that $W_{k}$ is an irreducible component of $\mathcal{V}\left(g_{k}\right)$.

Conversely, suppose that $W_{k}$ is an irreducible component of $\mathcal{V}\left(g_{k}\right)$. If $Y$ is an irreducible algebraic set such that $V \subset Y \subset X_{f}^{\infty}\left(\left\{d_{r}(f, V)\right\}_{r=0}^{\infty}\right)$, consider the irreducible algebraic set

$$
Y_{k}=\overline{\left\{y \in \mathcal{V}\left(g_{k}\right) \mid\left\{d_{r}\left(f, \pi_{k}(y)\right)\right\}_{r=0}^{\infty}=\left\{d_{r}(f, Y)\right\}_{r=0}^{\infty}\right\}} \subset \mathcal{V}\left(g_{k}\right)
$$

Since $W_{k}$ is an irreducible component of $\mathcal{V}\left(g_{k}\right)$ and $Y_{k}$ is an irreducible algebraic set such that $W_{k} \subset Y_{k} \subset \mathcal{V}\left(g_{k}\right)$, it follows that $W_{k}=Y_{k}$. In particular, we know that $V=\overline{\pi\left(W_{k}\right)}=\overline{\pi\left(Y_{k}\right)}=$ $Y$. Hence, $V$ is an irreducible component of $X_{f}^{\infty}\left(\left\{d_{r}(f, V)\right\}_{r=0}^{\infty}\right)$ yielding that $V$ is an isosingular set with respect to $f$.

Theorem 5.4 Every isosingular set is generically isomorphic to an irreducible and generically reduced component of a polynomial system constructed using deflation.

Proof. Lemma 5.3 yields that isosingular sets are generically isomorphic to an irreducible component of a polynomial system constructed using deflation. The statement now follows from Lemma 4.7 .

Theorem 5.4 yields that we can numerically represent isosingular sets using witness sets of projections [15.

The following corollary shows that isosingular sets are equivalently defined in terms of the limits of deflation sequences.

Corollary 5.5 If $f: \mathbb{C}^{N} \rightarrow \mathbb{C}^{n}$ is a polynomial system and $V \subset \mathcal{V}(f)$ is a nonempty irreducible algebraic set, then the following are equivalent.

1. $V$ is an isosingular set with respect to $f$.

2. $\lim _{k \rightarrow \infty} d_{k}(f, V)=\operatorname{dim} V$.

Proof. Since $V \neq \emptyset$, we can find $\widehat{x} \in V$ such that $V$ and $\widehat{x}$ have the same deflation sequence.

If $V$ is an isosingular set, by Theorem 5.4 there exists $k \geq 0$ such that if $\left(g_{k}, \widehat{y}_{k}\right)=\mathcal{D}^{k}(f, \widehat{x})$ and $W_{k}$ is defined by (14), then $W_{k}$ is an irreducible and generically reduced component of $\mathcal{V}\left(g_{k}\right)$ with $V=\overline{\pi_{k}\left(W_{k}\right)}$. In particular, $\lim _{\ell \rightarrow \infty} d_{\ell}\left(g_{k}, W_{k}\right)=d_{0}\left(g_{k}, W_{k}\right)=\operatorname{dim} W_{k}=\operatorname{dim} V$. Since $d_{j+k}(f, V)=d_{j}\left(g_{k}, W_{k}\right)$ for all $j \geq 0$, we have that $d_{k}(f, V) \rightarrow \operatorname{dim} V$.

Conversely, fix $k \geq 0$ such that $d_{k}(f, V)=\operatorname{dim} V$. For $\left(g_{k}, \widehat{y}_{k}\right)=\mathcal{D}^{k}(f, \widehat{x})$ and $W_{k}$ defined by (14), we know that $\operatorname{dim} W_{k}=\operatorname{dim} V=d_{k}(f, V)=d_{0}\left(g_{k}, W_{k}\right)$. This immediately yields that $W_{k}$ is an irreducible and generically reduced component of $\mathcal{V}\left(g_{k}\right)$ with $V=\overline{\pi_{k}\left(W_{k}\right)}$. Theorem 5.4 yields that $V$ is an isosingular set.

\subsection{Singular points of isosingular sets}

For generically reduced irreducible components, singular points are naturally defined in terms of the tangent space. We can extend this definition to isosingular sets using deflation sequences. With this, we will show that the number of distinct deflation sequences for a given polynomial system is finite and, in particular, the number of isosingular sets for a given polynomial system 
is finite. In particular, there is a finite bound on the maximum number of deflations needed to regularize every isosingular set for a given polynomial system.

The following defines the singular points of an isosingular set.

Definition 5.6 Let $f: \mathbb{C}^{N} \rightarrow \mathbb{C}^{n}$ be a polynomial system and $V$ be an isosingular set with respect to $f$. Then, the set of singular points of $V$ with respect to $f$ is the set

$$
\operatorname{Sing}_{f}(V)=\left\{x \in V \mid\left\{d_{r}(f, x)\right\}_{r=0}^{\infty} \neq\left\{d_{r}(f, V)\right\}_{r=0}^{\infty}\right\} .
$$

The set of smooth points of $V$ with respect to $f$ is the set

$$
V \backslash \operatorname{Sing}_{f}(V)=\left\{x \in V \mid\left\{d_{r}(f, x)\right\}_{r=0}^{\infty}=\left\{d_{r}(f, V)\right\}_{r=0}^{\infty}\right\} .
$$

Lemma 5.7 If $f: \mathbb{C}^{N} \rightarrow \mathbb{C}^{n}$ is a polynomial system and $V \subset \mathcal{V}(f)$ is an isosingular set with respect to $f$, then $\operatorname{Sing}_{f}(V)$ is a proper algebraic subset of $V$.

Proof. Since $V$ is an isosingular set, Corollary 5.5 yields that $\operatorname{dim} V=\lim _{\ell \rightarrow \infty} d_{\ell}(f, V)$. In particular, we can fix $k \geq 0$ such that $d_{j}(f, V)>\operatorname{dim} V$ for $0 \leq j<k$ and $d_{j}(f, V)=\operatorname{dim} V$ for $j \geq k$.

For each $\ell \geq 0$, consider the set

$$
S_{\ell}(V)=\left\{x \in V \mid d_{j}(f, x)=d_{j}(f, V) \text { for } 0 \leq j<\ell \text { and } d_{\ell}(f, x)>d_{\ell}(f, V)\right\} .
$$

Clearly, $S_{\ell}(V)$ is a proper algebraic subset of $V$ with $\operatorname{Sing}_{f}(V)=\bigcup_{\ell \geq 0} S_{\ell}(V)$. If $\ell>k$ and $x \in S_{\ell}(V)$, we know that $d_{j}(f, x)=d_{j}(f, V)$ for $0 \leq j \leq k$. In particular, using Lemma 3.4 we have

$$
\operatorname{dim} V=d_{k}(f, V)=d_{k}(f, x) \geq d_{\ell}(f, x)>d_{\ell}(f, V)=\operatorname{dim} V
$$

which is a contradiction. Thus, $S_{\ell}(V)=\emptyset$ for all $\ell>k$ yielding that $\operatorname{Sing}_{f}(V)$ is a finite union of proper algebraic subsets of $V$.

This definition of singular points agrees with the classical definition of singular points when $V$ is a generically reduced irreducible component of $\mathcal{V}(f)$. In this case, $\operatorname{dim} V=d_{k}(f, V)$ for all $k \geq 0$ and, for any $x \in V$,

$$
\left\{d_{r}(f, x)\right\}_{r=0}^{\infty} \neq\left\{d_{r}(f, V)\right\}_{r=0}^{\infty} \text { if and only if } \operatorname{dim} n u l l ~ J f(x)=d_{0}(f, x)>d_{0}(f, V)=\operatorname{dim} V .
$$

By Theorem 5.2. every isosingular set is generically isomorphic to a generically reduced irreducible component of some polynomial system created through deflation. The following shows that these sets are naturally related.

Lemma 5.8 Let $f: \mathbb{C}^{N} \rightarrow \mathbb{C}^{n}$ be a polynomial system, $V \subset \mathcal{V}(f)$ be an isosingular set, and $\widehat{x} \in V \backslash \operatorname{Sing}_{f}(V)$. Let $k \geq 1$ and $\left(g_{k}, \widehat{y}_{k}\right)=\mathcal{D}_{e}^{k}(f, \widehat{x})$, that is, $g_{k}$ is constructed using extrinsic strong deflation. If $z \in V$ such that $d_{0}(f, z)>d_{0}(f, V)$, then

$$
\operatorname{dim}\left(\pi_{k}^{-1}(z) \cap \mathcal{V}\left(g_{k}\right)\right) \geq d_{0}(f, V) .
$$

In particular, for every $w \in \pi_{k}^{-1}(z) \cap \mathcal{V}\left(g_{k}\right)$, $\operatorname{dnull}\left(g_{k}, w\right) \geq d_{0}(f, V)$. 
Proof. Let $d=d_{0}(f, V)$ and $\mathcal{L}: \mathbb{C}^{N} \rightarrow \mathbb{C}^{d}$ be a general linear system. Since $\operatorname{dnull}(f, z)=$ $d_{0}(f, z)>d$, we know that $\left[\begin{array}{c}J f(z) \cdot \eta \\ \mathcal{L}(\eta)\end{array}\right]=0$ defines a linear space of dimension $d_{0}(f, z)-d \geq 1$. In particular, it is clear from (13) that $g_{1}\left(z, \eta_{1}, \ldots, \eta_{d}\right)=0$ defines a linear space of dimension $d \cdot\left(d_{0}(f, z)-d\right) \geq d=d_{0}(f, V)$. That is, $\pi_{1}^{-1}(z) \cap \mathcal{V}\left(g_{1}\right)$ has dimension at least $d_{0}(f, V)$.

Suppose the lemma holds for $k \geq 1$. Fix $w \in \pi_{k}^{-1}(z) \cap \mathcal{V}\left(g_{k}\right)$. Since $w$ lies on some irreducible component of $\mathcal{V}\left(g_{k}\right)$ of dimension at least $d_{0}(f, V)$, we know that $\operatorname{dnull}\left(g_{k}, w\right) \geq d_{0}(f, V)$. In particular, since $\operatorname{dnull}\left(g_{k}, w\right) \geq d_{0}(f, V) \geq d_{k+1}(f, V)$, we know that that $g_{k+1}(w, \eta)=0$ has a solution. Since

$$
\pi_{k+1}^{-1}(z) \cap \mathcal{V}\left(g_{k+1}\right)=\left\{(w, \eta) \in \mathcal{V}\left(g_{k+1}\right) \mid w \in \pi_{k}^{-1}(z) \cap \mathcal{V}\left(g_{k}\right)\right\},
$$

it follows that $\pi_{k+1}^{-1}(z) \cap \mathcal{V}\left(g_{k+1}\right)$ also has dimension at least $d_{0}(f, V)$.

Theorem 5.9 Let $f: \mathbb{C}^{N} \rightarrow \mathbb{C}^{n}$ be a polynomial system, $V \subset \mathcal{V}(f)$ be an isosingular set, and $\widehat{x} \in V \backslash \operatorname{Sing}_{f}(V)$. Let $k \geq 0$ such that $W_{k} \subset \mathcal{V}\left(g_{k}\right)$ defined by (14) is a generically reduced irreducible component that is generically isomorphic to $V$ where $\left(g_{k}, y_{k}\right)=\mathcal{D}_{e}^{k}(f, \widehat{x})$. Then, for any $z \in V$, we have $z \in \operatorname{Sing}_{f}(V)$ if and only if, for any $\eta$ such that $(z, \eta) \in W_{k}$, $(z, \eta) \in \operatorname{Sing}_{g_{k}}\left(W_{k}\right)$.

Proof. If $z \in V \backslash \operatorname{Sing}_{f}(V)$, then $\left\{d_{r}(f, z)\right\}_{r=0}^{\infty}=\left\{d_{r}(f, V)\right\}_{r=0}^{\infty}$. Thus, there exists a unique $\eta$ such that $(z, \eta) \in W_{k}$. We know that

$$
\operatorname{dim} \text { null } J g_{k}(z, \xi)=d_{k}(f, z)=d_{k}(f, V)=d_{0}\left(g_{k}, W_{k}\right)=\operatorname{dim} W_{k}
$$

yielding $(z, \eta) \notin \operatorname{Sing}_{g_{k}}\left(W_{k}\right)$.

If $z \in \operatorname{Sing}_{f}(V)$, then $\left\{d_{r}(f, z)\right\}_{r=0}^{\infty} \neq\left\{d_{r}(f, V)\right\}_{r=0}^{\infty}$. Suppose that $\eta$ is such that $(z, \eta) \in W_{k}$. By construction of $k$, we know that there must exist $\ell \in\{0,1, \ldots, k\}$ such that $d_{j}(f, z)=d_{j}(f, V)$ for $0 \leq j<\ell$ and $d_{\ell}(f, z) \neq d_{\ell}(f, V)$. In particular, $d_{\ell}(f, z)>d_{\ell}(f, V)$. Since the case $\ell=k$ is trivial, we assume that $\ell<k$. By construction of $k$, we know $d_{\ell}(f, V)>\operatorname{dim} V$. Since $g_{\ell}$ only depends on the deflation sequence of $V$ up to $\ell-1$ and $d_{j}(f, z)=d_{j}(f, V)$ for $0 \leq j<\ell$, it follows that $\pi_{\ell}^{-1}(z) \cap \mathcal{V}\left(g_{\ell}\right)$ must consist of one point, namely $z_{\ell}$. Applying Lemma 5.8 using $g_{\ell}$, $W_{\ell}$, and $z_{\ell}$ yields that

$$
\operatorname{dnull}\left(g_{\ell},(z, \eta)\right) \geq d_{0}\left(g_{\ell}, W_{\ell}\right)=d_{\ell}(f, V)>\operatorname{dim} V=\operatorname{dim} W_{k} .
$$

Hence, $(z, \eta) \in \operatorname{Sing}_{g_{k}}\left(W_{k}\right)$.

The following theorem presents another description of isosingular sets.

Theorem 5.10 Let $f: \mathbb{C}^{N} \rightarrow \mathbb{C}^{n}$ be a polynomial system and $V \subset \mathcal{V}(f)$ be an isosingular set with respect to $f$. Then, either $V$ is an irreducible component of $\mathcal{V}(f)$ or there exists an isosingular set $U \subset \mathcal{V}(f)$ such that $V$ is an irreducible component of $\operatorname{Sing}_{f}(U)$.

Proof. Suppose that $V$ is an isosingular set with respect to $f$ such that $V$ is not an irreducible component of $\mathcal{V}(f)$. Thus, there exists an irreducible component $U \subset \mathcal{V}(f)$, which is an isosingular set with respect to $f$, such that $V \subsetneq U$. Hence, $\operatorname{dim} V<\operatorname{dim} U$ and, since $V$ is an isosingular set, we know $V \subset \operatorname{Sing}_{f}(U)$.

We have that $U$ is an isosingular set with respect to $f \operatorname{such}$ that $V \subset \operatorname{Sing}_{f}(U)$. If $V$ is not an irreducible component of $\operatorname{Sing}_{f}(U)$, then there exists an irreducible component $W \subset \operatorname{Sing}_{f}(U)$ 
such that $V \subsetneq W$. Hence, $\operatorname{dim} V<\operatorname{dim} W<\operatorname{dim} U$ and, since $V$ is an isosingular set, we know $V \subset \operatorname{Sing}_{f}(W)$.

We can repeat the same argument by renaming $W$ as $U$. Due to dimensionality, after finitely many times, we must have that $V$ is an irreducible component of the singular set of some isosingular set.

An immediate corollary of this theorem is that the number of isosingular sets is finite.

Corollary 5.11 For any polynomial system $f: \mathbb{C}^{N} \rightarrow \mathbb{C}^{n}$, the number of isosingular sets of $f$ is finite. In particular, the number of distinct deflation sequences is finite, that is,

$$
\left|\left\{\left\{d_{r}(f, x)\right\}_{r=0}^{\infty} \mid x \in \mathcal{V}(f)\right\}\right|<\infty .
$$

Proof. Let $d=\operatorname{dim} \mathcal{V}(f)$. Clearly, the number of isosingular sets of dimension $d$ is finite since each must be an irreducible component of $\mathcal{V}(f)$.

Suppose that, for $1 \leq k \leq d$, the number of isosingular sets of dimension at least $k$ is finite. Since every isosingular set of $f$ of dimension $k-1$ must either be an irreducible component of $\mathcal{V}(f)$ or an irreducible component of a singular set of an isosingular set of dimension at least $k$, the number of isosingular sets of dimension at least $k-1$ is also finite.

The previous theorem and corollary justify the following algorithm for computing every isosingular set of a polynomial system. For this to be a complete algorithm, we need an algorithm which determines if a given nonempty irreducible algebraic subset of $\mathcal{V}(f)$ is an isosingular set with respect to $f$, which is presented in Section 6 .

Algorithm 5.12 For a polynomial system $f: \mathbb{C}^{N} \rightarrow \mathbb{C}^{n}$, we will compute a set $\mathcal{I}$ which consists of the isosingular subsets of $\mathcal{V}(f)$. If $\mathcal{V}(f)=\emptyset$, then we return $\mathcal{I}=\emptyset$. Otherwise, we initialize $\mathcal{I}$ to be the set consisting of the irreducible components of $\mathcal{V}(f)$. For each $V \in \mathcal{I}$, compute the irreducible components of $\operatorname{Sing}_{f}(V)$ and add to $\mathcal{I}$ the ones which are isosingular sets. We keep performing this loop until no more elements are added to $\mathcal{I}$.

Example 5.13 Consider the Whitney umbrella defined by $f(x, y, z)=x^{2}-y^{2} z$. The real part of $\mathcal{V}(f)$ is displayed in Figure 1 .

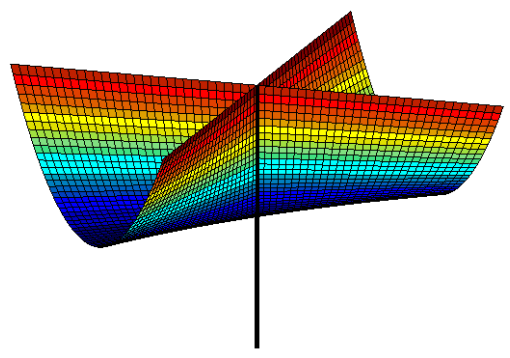

Figure 1: Whitney umbrella

Since $f$ is irreducible, $\mathcal{V}(f)$ is an irreducible surface which is generically reduced with deflation sequence $\{2,2,2, \ldots\}$. For $\alpha \in \mathcal{V}(f)$, we know that $\alpha \in \operatorname{Sing}_{f}(\mathcal{V}(f))$ if and only if $d_{0}(f, \alpha)=3$ 
which occurs if and only if the Jacobian of $f$ at $\alpha$ is identically zero. In particular, $\operatorname{Sing}_{f}(\mathcal{V}(f))=$ $\mathcal{V}(g)$ where

$$
g(x, y, z)=\left[\begin{array}{c}
x^{2}-y^{2} z \\
x \\
y z \\
y^{2}
\end{array}\right] .
$$

Clearly, $\mathcal{V}(g)=\{(0,0, z) \mid z \in \mathbb{C}\}$ which is often called the "handle" of the umbrella. Since $\operatorname{dim} \mathcal{V}(g)=1$ and the deflation sequence of $\mathcal{V}(g)$ with respect to $f$ is $\{3,1,1, \ldots\}, \mathcal{V}(g)$ is an isosingular set with respect to $f$. For a point $\alpha \in \mathcal{V}(g)$, we know that $\alpha \in \operatorname{Sing}_{f}(\mathcal{V}(g))$ if and only if $d_{1}(f, \alpha) \geq 2$ which occurs if and only if the Jacobian of $g$ at $\alpha$ has at least a two-dimensional null space. It is easy to verify that $\operatorname{Sing}_{f}(\mathcal{V}(g))=\{(0,0,0)\}$ and the deflation sequence of the origin with respect to $f$ is $\{3,2,0,0, \ldots\}$. In particular, the origin is an isosingular point with respect to $f$.

In summary, the isosingular sets of the Whitney umbrella are the irreducible surface $\mathcal{V}(f)$, the "handle" $\{(0,0, z) \mid z \in \mathbb{C}\}$, and the origin $\{(0,0,0)\}$. Hence, deflation at the origin restores quadratic convergence which is a markedly different behavior than blowing up at the origin which reproduces the singularity 18.

\subsection{Characterization of isosingular sets}

For a polynomial system $f$, Algorithm 5.12 presents one approach for computing every isosingular set with respect to a given polynomial system. This section characterizes which irreducible algebraic subsets of $\mathcal{V}(f)$ are isosingular sets. In particular, we want to construct a map Iso $f$ from the set of nonempty irreducible algebraic subsets of $\mathcal{V}(f)$ to the set of isosingular sets with respect to $f$. That is, if $V \subset \mathcal{V}(f)$ is a nonempty irreducible algebraic set, then $\operatorname{Iso}_{f}(V)$ will be the isosingular set with respect to $f$ containing $V$ such that $V$ and $\operatorname{Iso}_{f}(V)$ have the same deflation sequence with respect to $f$. Hence, generic points of $V$ are smooth points of $\operatorname{Iso}_{f}(V)$. By abuse of notation, for $x \in \mathcal{V}(f)$, define $\operatorname{Iso}_{f}(x)$ as $\operatorname{Iso}_{f}(\{x\})$. In this case, $x$ will be a smooth point of $\operatorname{Iso}_{f}(x)$ and it is the largest irreducible algebraic subset of $\mathcal{V}(f)$ containing $x$ for which $x$ is a smooth point.

The following lemma describes how to construct the map $\operatorname{Iso}_{f}$.

Lemma 5.14 Let $f: \mathbb{C}^{N} \rightarrow \mathbb{C}^{n}$ be a polynomial system and $V \subset \mathcal{V}(f)$ be a nonempty irreducible algebraic set. Then, there is a unique isosingular set $\operatorname{Iso}_{f}(V)$ with respect to $f$ containing $V$ such that $\operatorname{Iso}_{f}(V)$ and $V$ have the same deflation sequence with respect to $f$.

Proof. Since $V \subset X_{f}^{\infty}\left(\left\{d_{r}(f, V)\right\}_{r=0}^{\infty}\right)$, let $U \subset X_{f}^{\infty}\left(\left\{d_{r}(f, V)\right\}_{r=0}^{\infty}\right)$ be an irreducible component, and hence an isosingular set, such that $V \subset U$. Since general points in $V$ have the same deflation sequence as $U$, general points in $V$ are smooth points of $U$. This fact immediately yields uniqueness.

The following theorem characterizes isosingular sets.

Theorem 5.15 Let $f: \mathbb{C}^{N} \rightarrow \mathbb{C}^{n}$ be a polynomial system and $V \subset \mathcal{V}(f)$ be a nonempty irreducible algebraic set. Then, the following are equivalent.

1. $V$ is an isosingular set with respect to $f$.

2. For every isosingular set $U \subset \mathcal{V}(f)$ with respect to $f$ such that $V \subsetneq U, V \subset \operatorname{Sing}_{f}(U)$. 
3. For every irreducible algebraic set $U \subset \mathcal{V}(f)$ such that $V \subsetneq U, V$ and $U$ have different deflation sequences with respect to $f$, that is, $V \subset \operatorname{Sing}_{f}\left(\operatorname{Iso}_{f}(U)\right)$.

Proof. Let $V$ be an isosingular set and suppose that $U \subset \mathcal{V}(f)$ is an isosingular set such that $V \subsetneq U$. Since $\lim _{k \rightarrow \infty} d_{k}(f, V)=\operatorname{dim} V<\operatorname{dim} U=\lim _{k \rightarrow \infty} d_{k}(f, U), V$ and $U$ must have different deflation sequences. This immediately yields $V \subset \operatorname{Sing}_{f}(U)$.

If $V$ is not an isosingular set, then $U=\operatorname{Iso}_{f}(V)$ is an isosingular set such that $V \subsetneq U$ but $V$ is not contained in $\operatorname{Sing}_{f}(U)$.

The equivalence of Items 2 and 3 is clear.

Example 5.16 Consider $f(x, y, z)=x y z, V_{1}=\{(0,0, z) \mid z \in \mathbb{C}\}$, and $V_{2}=\{(0, z, z) \mid z \in \mathbb{C}\}$. It is easy to verify that the deflation sequences of $V_{1}$ and $V_{2}$, respectively, are

$$
\{3,1,1,1, \ldots\} \text { and }\{2,2,2,2, \ldots\} \text {. }
$$

In particular, Corollary 5.5yields that $V_{1}$ is an isosingular set of $f$ and $V_{2}$ is not. The irreducible algebraic set $U_{2}=\{(0, y, z) \mid y, z \in \mathbb{C}\}$ is an isosingular set of $f$ such that $U_{2}$ and $V_{2}$ have the same deflation sequence. In particular, $V_{2} \subsetneq \operatorname{Iso}_{f}\left(V_{2}\right)=U_{2}$.

The following corollary characterizes isosingular points which are the points for which the deflation sequence limits to 0 , i.e., deflation terminates. In particular, Item 4 shows that isosingular points are isolated in the sense that all nearby solutions have different deflation sequences.

Corollary 5.17 Let $f: \mathbb{C}^{N} \rightarrow \mathbb{C}^{n}$ be a polynomial system and $x \subset \mathcal{V}(f)$. Then, the following are equivalent.

1. $x$ is an isosingular point with respect to $f$.

2. For every isosingular set $U \subset \mathcal{V}(f)$ with respect to $f$ such that $\{x\} \subsetneq U, x \in \operatorname{Sing}_{f}(U)$.

3. For every irreducible algebraic set $U \subset \mathcal{V}(f)$ such that $\{x\} \subsetneq U, x$ and $U$ have different deflation sequences with respect to $f$, that is, $x \in \operatorname{Sing}_{f}\left(\operatorname{Iso}_{f}(U)\right)$.

4. There exists $\epsilon>0$ such that if $y \in \mathcal{V}(f)$ with $0<\|x-y\|<\epsilon$, then $x$ and $y$ have different deflation sequences with respect to $f$, that is, $\left\{d_{r}(f, x)\right\}_{r=0}^{\infty} \neq\left\{d_{r}(f, y)\right\}_{r=0}^{\infty}$.

Proof. The equivalence of Items 1,3 follows immediately from Theorem 5.15 .

Assume that Item 4 holds. If $\operatorname{Iso}_{f}(x)$ is positive dimensional, then there exists $y \in \operatorname{Iso}_{f}(x)$ such that $y$ and $\operatorname{Iso}_{f}(x)$ have the same deflation sequence and $0<\|x-y\|<\epsilon$. Since $x$ and $y$ have different deflation sequences, $x$ and $\operatorname{Iso}_{f}(x)$ have different deflation sequences, which is a contradiction. Hence, $\{x\}=\operatorname{Iso}_{f}(x)$ yielding that Item 1 holds.

Assume that Item 4 does not hold. Then, for every $\ell \geq 1$, we can find $y_{\ell} \in \mathcal{V}(f)$ such that $0<\left\|x-y_{\ell}\right\|<\frac{1}{\ell}$ where $x$ and $y_{\ell}$ have the same deflation sequence. Clearly, $x, y_{\ell} \in$ $X_{f}^{\infty}\left(\left\{d_{r}(f, x)\right\}_{r=0}^{\infty}\right)$ for all $\ell \geq 1$. Since $y_{\ell} \neq x$ and $y_{\ell} \rightarrow x,\{x\}$ is not an irreducible component of $X_{f}^{\infty}\left(\left\{d_{r}(f, x)\right\}_{r=0}^{\infty}\right)$ yielding that Item 1 does not hold.

\subsection{Isosingular local dimension}

Suppose that $f$ is a polynomial system and $x \in \mathcal{V}(f)$. Since $x$ and $\operatorname{Iso}_{f}(x)$ have the same deflation sequence and $\operatorname{Iso}_{f}(x)$ is an isosingular set, $d_{k}(f, x) \rightarrow \operatorname{dim} \operatorname{Iso}_{f}(x)$. That is, the deflation sequence of $x$ with respect to $f$ converges to the dimension of the largest irreducible algebraic subset of $\mathcal{V}(f)$ containing $x$ such that $x$ is a smooth point. We formally define this as the isosingular local dimension. 
Definition 5.18 For a polynomial system $f: \mathbb{C}^{N} \rightarrow \mathbb{C}^{n}$ and a point $x \in \mathcal{V}(f)$, the isosingular

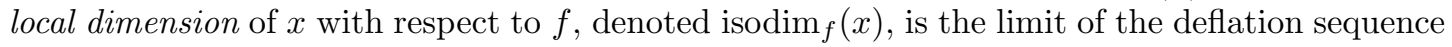
$\left\{d_{r}(f, x)\right\}_{r=0}^{\infty}$. In particular, $\operatorname{isodim}_{f}(x)=\operatorname{dim}_{\operatorname{Iso}_{f}}(x)$.

The local dimension of $x$ with respect to $\mathcal{V}(f)$, denoted $\operatorname{dim}_{f}(x)$, is the maximal dimension of the irreducible components of $\mathcal{V}(f)$ containing $x$. It is clear that

$$
\operatorname{dim}_{f}(x) \geq \operatorname{isodim}_{f}(x) .
$$

In particular, the isosingular local dimension is a lower bound on the local dimension. These values coincide if and only if $\operatorname{Iso}_{f}(x)$ is the unique irreducible component of $\mathcal{V}(f)$ containing $x$ which occurs if and only if $x$ is a smooth point on some (unique) irreducible component of $\mathcal{V}(f)$.

Example 5.19 Continuing with $f(x, y, z)=x y z$, consider $a_{0}=(0,0,0), a_{1}=(0,0,1)$ and $a_{2}=(0,1,1)$. It is easy to verify that

$$
\operatorname{Iso}_{f}\left(a_{0}\right)=\left\{a_{0}\right\}, \operatorname{Iso}_{f}\left(a_{1}\right)=\{(0,0, z) \mid z \in \mathbb{C}\} \text {, and } \operatorname{Iso}_{f}\left(a_{2}\right)=\{(0, y, z) \mid y, z \in \mathbb{C}\} .
$$

In particular, for $j=0,1,2, \operatorname{isodim}_{f}\left(a_{j}\right)=j$.

\section{Computing deflation sequences and isosingular sets}

For isolated solutions, deflation sequences must terminate and the number of deflations needed for termination is bounded by the depth. For isosingular sets in general, deflation sequences must stabilize, but a similar bound on the number of deflations is unknown. Obviously, a necessary condition for stabilization is that two consecutive terms in the deflation sequence must be equal, but this is not sufficient as clearly seen by considering the family of examples

$$
f_{k, \ell}(x, y)=\left[\begin{array}{l}
x^{k} \\
y^{\ell}
\end{array}\right]
$$

at the origin. In particular, if $1 \leq k<\ell$, one can verify that the deflation sequence at the origin with respect to $f_{k, \ell}$ is

$$
\{\overbrace{\underbrace{2, \ldots, 2}_{k-1}, 1, \ldots, 1}^{\ell-1}, 0,0, \ldots\} .
$$

We will present both a numerical and symbolic algorithm for determining that the deflation sequence has stabilized as well as both a numerical and symbolic algorithm for computing $\operatorname{Iso}_{f}(x)$ given $x \in \mathcal{V}(f)$. We remark that such an algorithm is sufficient to compute $\operatorname{Iso}_{f}(V)$ for any

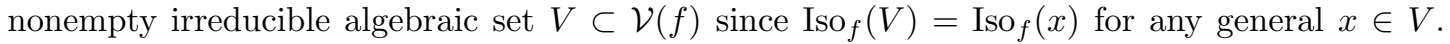
Since $V$ is an isosingular set with respect to $f$ if and only if $V=\operatorname{Iso}_{f}(V)$, which occurs if and only if $\operatorname{dim} V=\operatorname{dim} \operatorname{Iso}_{f}(V)$, such an algorithm can be used to determine if $V$ is an isosingular set.

We say that the deflation sequence $\left\{d_{r}(f, x)\right\}_{r=0}^{\infty}$ has stabilized for index $\ell \geq 0$ if $d_{\ell}(f, x)=$ $\lim _{k \rightarrow \infty} d_{k}(f, x)$. The deflation sequence at $x$ with respect to $f$ has stabilized at index $\ell$ if and only if, for $\left(g_{\ell}, y_{\ell}\right)=\mathcal{D}^{\ell}(f, x)$, the local dimension of $g_{\ell}$ at $y_{\ell}$ is $d_{\ell}(f, x)$, that is, $\operatorname{dim}_{g_{\ell}}\left(y_{\ell}\right)=$ dnull $\left(g_{\ell}, y_{\ell}\right)$. This occurs if and only if $y_{\ell}$ is a smooth point with respect to $g_{\ell}$ on a unique irreducible component of $\mathcal{V}\left(g_{\ell}\right)$ which is generically reduced of dimension $d_{\ell}(f, x)$. When using the determinantal form, this unique irreducible component is $\operatorname{Iso}_{f}(x)$, otherwise this irreducible 
component is generically isomorphic to $\operatorname{Iso}_{f}(x)$. Moreover, when using the determinantal form of deflation, which is amenable to symbolic computations, the coefficients of each deflation system are in the same subfield of $\mathbb{C}$ as the coefficients of the original system.

The previous paragraph is used to help justify the algorithms in this section. Moreover, to simplify the presentation of the algorithms, we will simply describe the key computation which is, for a polynomial system $f: \mathbb{C}^{N} \rightarrow \mathbb{C}^{n}$ and $x \in \mathcal{V}(f)$, to determine if $\operatorname{dim}_{f}(x)=\operatorname{dnull}(f, x)$.

Algorithm 6.1 For a polynomial system $f: \mathbb{C}^{N} \rightarrow \mathbb{C}^{n}$ and a point $x \in \mathcal{V}(f)$, we will determine if $\operatorname{dim}_{f}(x)=\operatorname{dnull}(f, x)$. If $\operatorname{dnull}(f, x)=0$, then return yes. Otherwise, compute the associated primes of dimension dnull $(f, x)$ for the ideal generated by $f$ (see 9 for more details). If any such prime ideal vanishes at $x$, then return yes, otherwise, return no.

Example 6.2 Reconsider the Whitney umbrella from Example 5.13 defined by $f(x, y, z)=$ $x^{2}-y^{2} z$ at $\alpha=(0,0,0)$. Since $2=\operatorname{dim}_{f}(\alpha)<d_{0}(f, \alpha)=3$, we immediately know the deflation sequence has not stabilized at index 0 . We have $\left(g_{1}, \alpha\right)=\mathcal{D}_{\text {det }}(f, \alpha)$ where $g_{1}$ is as in (18). One can verify that $\mathcal{V}\left(g_{1}\right)$ is irreducible, generically reduced, and one dimensional yielding that $1=\operatorname{dim}_{g_{1}}(\alpha)<d_{1}(f, \alpha)=2$. Hence, the deflation sequence has not stabilized at index 1 . If $\left(g_{2}, \alpha\right)=\mathcal{D}_{\operatorname{det}}^{2}(f, \alpha)$, one can verify that the ideal generated by $g_{2}$ is $\langle x, y, z\rangle$ yielding that deflation has stabilized at index 2 with $d_{2}(f, \alpha)=0$ and $\operatorname{Iso}_{f}(\alpha)=\{\alpha\}=\{(0,0,0)\}$.

We now consider the corresponding numerical approaches. We note that even if we only have a numerical approximation of the solution $x$, the point $x$ is used to generate nonnegative integers, namely the deflation sequence with the deflation process being a symbolic operation based solely on the deflation sequence. In particular, we assume that we know a sufficiently accurate approximation of $x$ to correctly identify the deflation sequence. If $x$ is the endpoint of a homotopy path, we can use endgames (see [19, 25, 26, 27, 32]) to compute $x$ to arbitrary accuracy.

Algorithm 6.3 For a polynomial system $f: \mathbb{C}^{N} \rightarrow \mathbb{C}^{n}$ and a point $x \in \mathcal{V}(f)$, we will determine if $\operatorname{dim}_{f}(x)=\operatorname{dnull}(f, x)$. If $\operatorname{dnull}(f, x)=0$, return yes. Otherwise, fix $d=\operatorname{dnull}(f, x)$ and let $\mathcal{L}: \mathbb{C}^{N} \rightarrow \mathbb{C}^{d}$ be a system of random linear polynomials such that $x \in \mathcal{V}(\mathcal{L})$. Let $A \in \mathbb{C}^{(N-d) \times n}$ and $\lambda \in \mathbb{C}^{d}$ be random and consider the polynomial system $\mathcal{R}(f): \mathbb{C}^{N} \rightarrow \mathbb{C}^{N-d}$ defined by $\mathcal{R}(f)=A \cdot f$ and homotopy

$$
H(y, t)=\left[\begin{array}{c}
\mathcal{R}(f)(y) \\
\mathcal{L}(y)+(1-t) \lambda
\end{array}\right]
$$

Since, with probability one, $x \in \mathcal{V}(\mathcal{R}(f))$ with $\operatorname{dnull}(\mathcal{R}(f), x)=d, x$ is a smooth point on some (unique) irreducible component, say $V \subset \mathcal{V}(\mathcal{R}(f)$ ), of dimension $d$. In particular, it follows that $H(x, 1)=0$ and $J_{y} H(x, 1)$ has rank $N$ yielding that we can use continuation starting at $x$ to compute a point $z \in V \cap \mathcal{V}(\mathcal{L}+\lambda)$. If $z \in \mathcal{V}(f)$, then return yes, otherwise return no.

Since $z$ is an isolated nonsingular root of the square system $\left[\begin{array}{c}\mathcal{R}(f) \\ \mathcal{L}+\lambda\end{array}\right]$, we can compute $z$ to arbitrary accuracy using Newton's method which means that the test $z \in \mathcal{V}(f)$ can be done reliably. Additionally, since computing such a $z$ is usually computationally inexpensive, we can perform this test multiple times using different $\mathcal{L}$ and $\lambda$ to enhance reliability. 
Example 6.4 Consider the polynomial $g_{1}$ from Example 6.2 which is defined in (18). For simplicity, consider

$$
A=\left[\begin{array}{llll}
6 & 8 & -7 & 4
\end{array}\right], \quad \mathcal{L}(x, y, z)=\left[\begin{array}{c}
2 x-4 y+6 z \\
7 x-8 y-9 z
\end{array}\right], \quad \text { and } \quad \lambda=\left[\begin{array}{c}
1+i \\
2-i
\end{array}\right]
$$

where $i=\sqrt{-1}$. Starting at $\alpha=(0,0,0)$ with the homotopy $H$ defined by $\sqrt{19})$, we obtained, to four digits, the point

$$
\beta=(-0.0257-0.0394 i, 0.2317+0.0076 i,-0.0037-0.1485 i) .
$$

Since $\beta$ clearly does not numerically vanish on $g_{1}$, we know that the deflation sequence of $\alpha$ with respect to $f(x, y, z)=x^{2}-y^{2} z$ has not stabilized at index 1. As shown in Example 6.2 . $d_{2}(f, \alpha)=0$ meaning that the deflation sequence of $\alpha$ with respect to $f$ stabilizes at index 2 .

Additionally, consider $\gamma=(0,0,1)$ for which $\operatorname{dnull}\left(g_{1}, \gamma\right)=1$. With

$$
A=\left[\begin{array}{cccc}
6 & 8 & -7 & 4 \\
-3 & 4 & -1 & 5
\end{array}\right], \quad \mathcal{L}(x, y, z)=2 x-4 y+6 z-6, \quad \text { and } \quad \lambda=1+i,
$$

consider the homotopy $H$ defined by 19 with start point $\gamma$. We obtained, to four digits, the point

$$
\delta=(0,0,0.8333-0.1667 i)
$$

which does numerically vanish on $g_{1}$. Hence, the deflation sequence of $\gamma$ with respect to $f$ has stabilized at index 1 .

After we have found that the deflation sequence has stabilized, we have a smooth point on a generically reduced and irreducible algebraic set. Using random monodromy loops 30 together with a linear trace test [31, we can compute a witness set for $\operatorname{Iso}_{f}(x)$ (see [15, 32] for more details). Theoretically, the expected number of random monodromy loops may be infinite but, in practice, only a small number of random loops are needed. Since the linear trace test determines if we have indeed computed a witness set, we obtain a clear signal of success.

\section{$7 \quad$ Examples}

We begin with a simple illustrative example, and then report on a more complicated example taken from kinematics.

\subsection{Simple example}

Consider the polynomial $x y z^{2}$. It is easy to see that the solution set $\mathcal{V}\left(x y z^{2}\right)$ factors into the three coordinate planes: $\mathcal{V}(x)$ and $\mathcal{V}(y)$ appear with generic multiplicity 1 , while the plane $\mathcal{V}(z)$ appears as the double plane $\mathcal{V}\left(z^{2}\right)$. Using the determinantal form of deflation, one can find by hand calculation that $\mathcal{V}\left(x y z^{2}\right)$ contains seven isosingular sets. These sets and their deflation sequences are:

- Planes $\mathcal{V}(x)$ and $\mathcal{V}(y),\{2,2, \ldots\}$

- Plane $\mathcal{V}(z),\{3,2,2, \ldots\}$

- Line $\mathcal{V}(x, y),\{3,1,1, \ldots\}$ 
- Lines $\mathcal{V}(x, z)$ and $\mathcal{V}(y, z),\{3,3,1,1, \ldots\}$;

- Point $\mathcal{V}(x, y, z),\{3,3,3,0,0, \ldots\}$.

The isosingular sets are finite in number and for each one, the deflation sequence stabilizes to the dimension of the set. After stabilization, each isosingular set is a reduced component of the associated deflated system.

\subsection{Foldable Stewart-Gough platform}

Stewart-Gough platforms are a kind of robotic device in which a moving end-plate is supported from a stationary base-plate by six prismatic (i.e., telescoping) legs. Each leg is attached to the base-plate at one end by a spherical (ball-and-socket) joint and is attached at the other end to the end-plate again by a spherical joint. The end-plate is moved through space by extending and contracting the six legs. For a general design, when the leg lengths are locked, the end-plate becomes immobile, that is, the system of kinematical equations has only isolated roots.

Griffis-Duffy platforms [12] are special Stewart-Gough platforms wherein the base- and endplates are triangles, with legs that connect a vertex to a side or a side to a vertex cyclically around the triangles. Husty and Karger [20] noticed that certain Griffis-Duffy platforms move with one degree of freedom with the leg lengths locked. A very special case in this family is a foldable Stewart-Gough platform, first studied in 24], in which the triangles are congruent, the joints on the sides of the triangles are at the midpoints, and the leg lengths are all equal to the altitude of the triangles. For easy reference, the equations are listed in Appendix A. In [24, it is shown that the solution set consists of a not physically meaningful surface and 22 one-dimensional components: twelve lines, three double lines, three quadrics, and four quartics. Of these, the three double lines, the three quadrics, and one of the quartics each contains a single connected real curve, while the rest contain only non-real complex points.

In [24, it was found that the real curves had four points of special interest. Three of these, the "folded out" configurations, are points where one of the double lines meets two of the quadrics. At the fourth point, the "folded in" configuration, all three double lines meet a self-crossing of the quartic curve. In [33], numerical evidence was presented showing that the folded in configuration has multiplicity 8 , which is consistent with the number of curve segments meeting there (three double lines and two arcs of the quartic).

With the isosingular theory presented here, we can compute all singular points on the solution curves of the foldable Stewart-Gough platform.

For our computations, we used the software package Bertini v1.2 [6], choosing the regenerative cascade [17] with adaptive precision tracking [4, 5, 7, to compute the numerical irreducible decompositions. The serial computations used a $2.4 \mathrm{GHz}$ Opteron 250 processor with 64 -bit Linux. The parallel computations ran on a cluster consisting of a manager that uses one core of a Xeon 5410 processor and 8 computing nodes each containing two $2.33 \mathrm{GHz}$ quad-core Xeon 5410 processors running 64 -bit Linux, i.e., one manager and 64 workers.

Let $L: \mathbb{C}^{4} \rightarrow \mathbb{C}$ be a general linear polynomial. Then, $L\left(e_{1}, e_{2}, e_{3}, e_{4}\right)=0$ defines an affine set for which the natural projection $\mathbb{C}^{4} \backslash\{(0,0,0,0)\} \rightarrow \mathbb{P}^{3}$ is one-to-one and onto a general coordinate patch $\mathbb{C}^{3} \subset \mathbb{P}^{3}$. We then solved

$$
\mathcal{F}=\left\{f_{1}, \ldots, f_{5}, f_{7}, L\right\}=0,
$$

where the $f_{i}$ are as in the Appendix $\mathrm{A}$. Since $f_{6}$ is contained the linear span of $f_{1}, \ldots, f_{5}$, we removed $f_{6}$ from the system. Additionally, we worked implicitly on the linear space defined by $\mathcal{V}(L)$. 
We used Bertini to compute a numerical irreducible decomposition of $\mathcal{V}(\mathcal{F})$ in 8.6 seconds using serial processing and found 22 curves, 19 of which have multiplicity one and three have multiplicity 2. In particular, as expected, there are 15 lines of which three are double lines, three quadrics, and four quartics. The deflation sequence for each multiplicity one curve is $\{1,1, \ldots\}$ and for each double line is $\{2,1,1, \ldots\}$, which is in agreement with Lemma 4.7 .

We then used Bertini to compute a numerical irreducible decomposition of

$$
\left\{x \in \mathcal{V}(\mathcal{F}) \mid d_{0}(\mathcal{F}, x) \geq 2\right\}
$$

using the fact that $d_{0}(\mathcal{F}, x) \geq 2$ if and only if the rows of $J \mathcal{F}(x)$ are linearly dependent. We choose this formulation since this reduces the number of variables and polynomials following Remarks 2.1 and 4.1. This computation took 36.5 seconds in parallel and yielded the isosingular point $x_{1}=(0,0,0,1 ; 0,0,0,0) \in \mathbb{P}^{3} \times \mathbb{C}^{4}$ which has deflation sequence $\{4,0,0, \ldots\}$, three isosingular points with deflation sequence $\{3,0,0, \ldots\}, 18$ isosingular points with deflation sequence $\{2,0,0, \ldots\}$, and the three double lines. The point $x_{1}$ is the folded-in pose and the three points with deflation sequence $\{3,0,0, \ldots\}$ are the folded-out configurations [24].

We also used Bertini to compute a numerical irreducible decomposition of

$$
\left\{x \in \mathcal{V}(\mathcal{F}) \mid d_{0}(\mathcal{F}, x) \geq 3\right\}
$$

using the fact that $d_{0}(\mathcal{F}, x) \geq 3$ if and only if the left null space of $J \mathcal{F}(x)$ is at least two dimensional. This computation took nearly 5 minutes in parallel and yielded the isosingular point $x_{1}$ with deflation sequence $\{4,0,0, \ldots\}$ and the three isosingular points with deflation sequence $\{3,0,0, \ldots\}$ computed above.

We have two types of curves, and we must consider if any of them might contain isosingular points that we have not already found. The possibilities are tightly constrained by the fact that any deflation sequence must be monotonic decreasing and the condition that an isosingular set inside a bigger one must at some stage have a higher entry in its deflation sequence than its containing set. This implies that anything inside of the curves with deflation sequence $\{1,1, \ldots\}$ must already have been found when we looked for points $x \in \mathcal{V}(\mathcal{F})$ such that $d_{0}(\mathcal{F}, x) \geq 2$. This leaves the double lines, which have deflation sequence $\{2,1,1, \ldots\}$. These double lines could contain points $x \in \mathcal{V}(\mathcal{F})$ such that $d_{0}(\mathcal{F}, x)=d_{1}(\mathcal{F}, x)=2$. By restricting computation to each of the three double lines, and imposing the condition $d_{1}(\mathcal{F}, x) \geq 2$, we computed four points on each double line with each computation taking roughly 90 seconds in parallel. Each of these 12 isosingular points have deflation sequence $\{2,2,0,0, \ldots\}$ and lie at the intersection of a double line with another component.

\section{Conclusions}

We have presented new concepts for studying singularities of algebraic sets, namely deflation sequences and isosingular sets. An isosingular set is a nonempty irreducible algebraic set for which the deflation sequence stabilizes to its dimension. In this case, deflation constructs a polynomial system which has a generically reduced irreducible component that is generically isomorphic to the given isosingular set. Since deflation is a symbolic operation given the null space dimension, we present two algorithms for computing isosingular sets, one which is symbolic and the other is symbolic-numeric. In particular, for a given polynomial system, the number of isosingular sets and distinct deflation sequences are finite and computable.

Additionally, given a polynomial system and any nonempty irreducible algebraic subset of its solution set, there is a unique isosingular set which contains the given set such that general 
points of the given set are smooth points of the isosingular set. In particular, isosingular theory presents a stratification of the singularities of the given polynomial system such that, for every solution, there is a unique isosingular set for which the given point is a smooth point on the isosingular set.

\section{Acknowledgments}

The authors would like to thank Gert-Martin Greuel for sharing his knowledge regarding ThomBoardman singularities and the Mittag-Leffler Institute (Djursholm, Sweden) for their support and hospitality when completing this article.

\section{References}

[1] V.I. Arnol'd. Singularities of smooth mappings. Russian Math. Surveys 23, 1-43, 1968.

[2] D.J. Bates, J.D. Hauenstein, C. Peterson, and A.J. Sommese. Numerical decomposition of the rank-deficiency set of a matrix of multivariate polynomials. In Approximate commutative algebra, Texts Monogr. Symbol. Comput., pages 55-77. Springer, Vienna, 2009.

[3] D.J. Bates, J.D. Hauenstein, C. Peterson, and A.J. Sommese. A numerical local dimension test for points on the solution set of a system of polynomial equations. SIAM J. Numer. Anal. 47(5), 3608-3623, 2009.

[4] D.J. Bates, J.D. Hauenstein, and A.J. Sommese. Efficient path tracking methods. To appear in Numer. Algorithms.

[5] D.J. Bates, J.D. Hauenstein, A.J. Sommese, and C.W. Wampler. Adaptive multiprecision path tracking. SIAM J. Numer. Anal., 46(2), 722-746, 2008.

[6] D.J. Bates, J.D. Hauenstein, A.J. Sommese, and C.W. Wampler. Bertini: Software for Numerical Algebraic Geometry. Available at http://www.nd.edu/ sommese/bertini.

[7] D.J. Bates, J.D. Hauenstein, A.J. Sommese, and C.W. Wampler. Stepsize control for adpative multiprecision path tracking. Contemp. Math., 496, 21-31, 2009.

[8] J. Boardman. Singularities of differentiable maps. Inst. Hautes Études Sci. Publ. Math. $33,21-57,1967$.

[9] D. Eisenbud. Commutative algebra with a view toward algebraic geometry. Graduate Texts in Mathematics, 150. Springer-Verlag, New York, 1995.

[10] B.H. Dayton and Z. Zeng. Computing the multiplicity structure in solving polynomial systems. In ISSAC'05, pages 116-123. ACM, New York, 2005.

[11] W. Fulton. Intersection theory, Second edition. Springer-Verlag, Berlin, 1998.

[12] M. Griffis and J. Duffy. Method and apparatus for controlling geomtrically simple parallel mechanisms with distinctive connections. US Patent 5,179,525, 1993.

[13] A. Griewank and M.R. Osborne. Analysis of Newton's method at irregular singularities. SIAM J. Numer. Anal. 20(4), 747-773, 1983. 
[14] J. Guckenheimer and Y. Xiang. Defining equations for bifurcations and singularities. Moscow Math. J. 3(3), 935-946, 2003.

[15] J.D. Hauenstein and A.J. Sommese. Witness sets of projections. Appl. Math. Comput. 217(7), 3349-3354, 2010.

[16] J.D. Hauenstein, A.J. Sommese, and C.W. Wampler. Regeneration homotopies for solving systems of polynomials. Math. Comp. 80, 345-377, 2011.

[17] J.D. Hauenstein, A.J. Sommese, and C.W. Wampler. Regenerative cascade homotopies for solving polynomial systems. Preprint, 2010. Available at http://www.math.tamu. edu/ jhauenst/preprints.

[18] H. Hauser. Seventeen obstacles for resolution of singularities. In Singularities (Oberwolfach, 1996), volume 162 of Progr. Math., pages 289-313. Birkhäuser, Basel, 1998.

[19] B. Huber and J. Verschelde. Polyhedral end games for polynomial continuation. Numer. Algorithms 18(1), 91-108, 1998.

[20] M.L. Husty and A. Karger. Self-motions of Griffis-Duffy type parallel manipulators. Proc. 2000 IEEE Int. Conf. Robotics and Automation, CDROM, San Francisco, CA, April 24-28, 2000.

[21] G. LeCerf. Quadratic Newton iteration for systems with multiplicity. Found. Comp. Math. 2(3), 247-293, 2002.

[22] A. Leykin, J. Verschelde, and A. Zhao. Newton's method with deflation for isolated singularities of polynomial systems. Theor. Comp. Sci. 359, 111-122, 2006.

[23] A. Leykin, J. Verschelde, and A. Zhao. Higher-order deflation for polynomial systems with isolated singular solutions. In Algorithms in algebraic geometry, volume 146 of IMA Vol. Math. Appl., pages 79-97. Springer, New York, 2008.

[24] Y. Lu, D.J. Bates, A.J. Sommese, and C.W. Wampler. Finding all real points of a complex curve. Contemp. Math., 448, 183-205, 2007.

[25] A.P. Morgan, A.J. Sommese, and C.W. Wampler. Computing singular solutions to nonlinear analytic systems. Numerische Math. 58, 669-684, 1991.

[26] A.P. Morgan, A.J. Sommese, and C.W. Wampler. A power series method for computing singular solutions to nonlinear analytic systems. Numerische Math. 63, 391-409, 1992.

[27] A.P. Morgan, A.J. Sommese, and C.W. Wampler. Computing singular solutions to polynomial systems. Adv. in Appl. Math. 13, 305-327, 1992.

[28] T. Ojika. Modified deflation algorithm for the solution of singular problems. I. A system of nonlinear algebraic equations. J. Math. Anal. Appl. 123, 199-221, 1987.

[29] T. Ojika, S. Watanabe, and T. Mitsui. Deflation algorithm for the multiple roots of a system of nonlinear equations. J. Math. Anal. Appl. 96, 463-479, 1983.

[30] A.J. Sommese, J. Verschelde and C.W. Wampler. Numerical decomposition of the solution sets of polynomial systems into irreducible components. SIAM J. Numer. Anal. 38(6), 2022-2046, 2001. 
[31] A.J. Sommese, J. Verschelde, and C.W. Wampler. Symmetric functions applied to decomposing solution sets of polynomial systems. SIAM J. Numer. Anal., 40(6), 2026-2046, 2002.

[32] A.J. Sommese and C.W. Wampler. The Numerical Solution of Systems of Polynomials Arising in Engineering and Science. World Scientific Press, Singapore, 2005.

[33] C.W. Wampler, J.D. Hauenstein, and A.J. Sommese. Mechanism mobility and a local dimension test. To appear in Mech. Machine Theory, 2011.

\section{A Foldable Stewart-Gough platform system}

The polynomials for the foldable Stewart-Gough platform, which are presented using the variables $e_{1}, e_{2}, e_{3}, e_{4}, g_{1}, g_{2}, g_{3}, g_{4}$, are as follows. If $r=\sqrt{3}$ and $G=g_{1}^{2}+g_{2}^{2}+g_{3}^{2}+g_{4}^{2}$, the polynomial system is $\left\{f_{1}, \ldots, f_{7}\right\}$ defined as

$$
\begin{aligned}
f_{1}=- & (2 / 3) e_{1} e_{1}-(4 r / 3) e_{2} e_{1}-2 e_{2} e_{2}-(8 / 3) e_{3} e_{3}-(r / 3) e_{1} g_{3}+3 e_{1} g_{4}-e_{2} g_{3}-r e_{2} g_{4} \\
& +(r / 3) e_{3} g_{1}+e_{3} g_{2}-3 e_{4} g_{1}+r e_{4} g_{2}+G \\
f_{2}=- & (2 / 3) e_{1} e_{1}+(4 r / 3) e_{2} e_{1}-2 e_{2} e_{2}-(8 / 3) e_{3} e_{3}-(r / 3) e_{1} g_{3}+3 e_{1} g_{4}+e_{2} g_{3}+r e_{2} g_{4} \\
& +(r / 3) e_{3} g_{1}-e_{3} g_{2}-3 e_{4} g_{1}-r e_{4} g_{2}+G \\
f_{3}=- & (8 / 3) e_{1} e_{1}-(8 / 3) e_{3} e_{3}+(2 r / 3) e_{1} g_{3}+(2 r) e_{2} g_{4}-(2 r / 3) e_{3} g_{1}-(2 r) e_{4} g_{2}+G \\
f_{4}=- & (2 / 3) e_{1} e_{1}-(4 r / 3) e_{2} e_{1}-2 e_{2} e_{2}-(8 / 3) e_{3} e_{3}-(r / 3) e_{1} g_{3}-3 e_{1} g_{4}-e_{2} g_{3}+r e_{2} g_{4} \\
& +(r / 3) e_{3} g_{1}+e_{3} g_{2}+3 e_{4} g_{1}-r e_{4} g_{2}+G \\
f_{5}=- & (2 / 3) e_{1} e_{1}+(4 r / 3) e_{2} e_{1}-2 e_{2} e_{2}-(8 / 3) e_{3} e_{3}-(r / 3) e_{1} g_{3}-3 e_{1} g_{4}+e_{2} g_{3}-r e_{2} g_{4} \\
& +(r / 3) e_{3} g_{1}-e_{3} g_{2}+3 e_{4} g_{1}+r e_{4} g_{2}+G \\
f_{6}=- & (8 / 3) e_{1} e_{1}-(8 / 3) e_{3} e_{3}+(2 r / 3) e_{1} g_{3}-(2 r) e_{2} g_{4}-(2 r / 3) e_{3} g_{1}+(2 r) e_{4} g_{2}+G \\
f_{7}=e_{1} & g_{1}+e_{2} g_{2}+e_{3} g_{3}+e_{4} g_{4}
\end{aligned}
$$

